\title{
Suppressors of Cytokine Signaling (SOCS)1 and SOCS3 Proteins Are Mediators of Interleukin-10 Modulation of Inflammatory Responses Induced by Chlamydia muridarum and Its Major Outer Membrane Protein (MOMP) in Mouse J774 Macrophages
}

\author{
Skyla A. Duncan, Rajnish Sahu, Saurabh Dixit ${ }^{\mathbb{D}}$, Shree R. Singh, and Vida A. Dennis \\ Center for NanoBiotechnology Research (CNBR), Department of Biological Sciences, Alabama State University, 1627 Harris Way, \\ Montgomery, AL 36104, USA
}

Correspondence should be addressed to Vida A. Dennis; vdennis@alasu.edu

Received 13 March 2020; Accepted 10 April 2020; Published 24 June 2020

Academic Editor: Mirella Giovarelli

Copyright (c) 2020 Skyla A. Duncan et al. This is an open access article distributed under the Creative Commons Attribution License, which permits unrestricted use, distribution, and reproduction in any medium, provided the original work is properly cited.

\begin{abstract}
The immunopathology of chlamydial diseases is exacerbated by a broad-spectrum of inflammatory mediators, which we reported are inhibited by IL-10 in macrophages. However, the chlamydial protein moiety that induces the inflammatory mediators and the mechanisms by which IL-10 inhibits them are unknown. We hypothesized that Chlamydia major outer membrane protein (MOMP) mediates its disease pathogenesis, and the suppressor of cytokine signaling (SOCS) 1 and SOCS3 proteins are mediators of the IL-10 inhibitory actions. Our hypothesis was tested by exposing mouse J774 macrophages to chlamydial stimulants (live Chlamydia muridarum and MOMP) with and without IL-10. MOMP significantly induced several inflammatory mediators (IL-6, IL-12p40, CCL5, CXCL10), which were dose-dependently inhibited by IL-10. Chlamydial stimulants induced the mRNA gene transcripts and protein expression of SOCS1 and SOCS3, with more SOCS3 expression. Notably, IL-10 reciprocally regulated their expression by reducing SOCS1 and increasing SOCS3. Specific inhibitions of MAPK pathways revealed that $\mathrm{p} 38, \mathrm{JNK}$, and MEK1/2 are required for inducing inflammatory mediators as well as SOCS1 and SOCS3. Chlamydial stimulants triggered an M1 pro-inflammatory phenotype evidently by an enhanced nos2 (M1 marker) expression, which was skewed by IL-10 towards a more M2 anti-inflammatory phenotype by the increased expression of mrc1 and arg1 (M2 markers) and the reduced SOCS1/SOCS3 ratios. Neutralization of endogenously produced IL-10 augmented the secretion of inflammatory mediators, reduced SOCS3 expression, and skewed the chlamydial M1 to an M2 phenotype. Inhibition of proteasome degradation increased TNF but decreased IL-10, CCL5, and CXCL10 secretion by suppressing SOCS1 and SOCS3 expressions and dysregulating their STAT1 and STAT3 transcription factors. Our data show that SOCS1 and SOCS3 are regulators of IL-10 inhibitory actions, and underscore SOCS proteins as therapeutic targets for IL-10 control of inflammation for Chlamydia and other bacterial inflammatory diseases.
\end{abstract}

\section{Introduction}

Chlamydia is one of the most prevalent bacterial sexually transmitted infections (STIs) worldwide, with an estimated annual incidence of 1.7 million cases in the United States [1]. The pathogenic agent responsible for this infection is Chlamydia trachomatis, a gram-negative and intracellular anaerobe bacterium [2] that causes mucosal infections of the genital, anorectal, and oropharyngeal surfaces in humans
[3]. Genital Chlamydia is associated with significant reproductive morbidity, including tubal factor infertility, with women being more disproportionately affected than men $[4,5]$.

The unique developmental cycle of $C$. trachomatis allows for its intracellular reproduction while infecting neighboring cells, resulting in persistent disease or re-infection even after treatment $[3,6,7]$. C. trachomatis also possesses diverse virulence factors including, its major outer membrane protein (MOMP) that exhibits high immunogenic potential $[8,9]$. 
We have published that MOMP or its peptide derivative encapsulated within biodegradable polymeric nanoparticles such as chitosan [10], PLGA [poly (D, L-lactide-co-glycolide)] [11] and PLA-PEG [poly(lactic acid)-poly(ethylene glycol)] [12], hold promise as Chlamydia nanovaccine candidates since they potentiated and enhanced adaptive immune responses. Notwithstanding, other researchers have reported that C. trachomatis infection induces overproduction of a variety of inflammatory cytokines (Interleukin-6 (IL-6), Interleukin-8 (IL-8), Interleukin-12p40 (IL-12p40)), Tumor necrosis factor (TNF), Growth-regulated oncogene-alpha $(\mathrm{GRO} \alpha)$, Granulocyte-macrophage colony-stimulating factor (GM-CSF) [13-16] and chemokines (Chemokine (C-C motif) ligand 5 (CCL5), Chemokine interferon- $\gamma$ inducible protein $10 \mathrm{kDa}(\mathrm{CXCL} 10))[15,17,18]$, which are implicated in chlamydial immunopathology $[6,19]$. During acute infection, C. trachomatis induces these inflammatory mediators to diminish the host immune response [20], and as the infection prolongs or repeated infections occur, more inflammatory mediators and immune cells are released to combat the infection $[21,22]$. Excessive production of inflammatory mediators contributes significantly to the disease manifestation by damaging neighboring cells $[23,24]$. Such observations confirm an intimate relationship between Chlamydia and the host immune system. In particular, these studies suggest that the ability of Chlamydia to hijack the immune response can account for some of the complicated pathologies associated with chlamydial diseases.

On the other hand, despite having a low mortality rate, $C$. trachomatis causes serious [22] complications that result in irreversible damage to the infected population if left untreated, thus becoming a considerable burden among high-risk people [2]. The incidence of C. trachomatis infections continues to rise despite over two decades of national screening efforts in the United States. Notably, antibiotics against Chlamydia is very effective; however, due to the asymptomatic nature of the disease, antibiotic treatment is inadequate in an already established and persistent infection [25]. Consequently, the development of an effective treatment would be invaluable for reducing the worldwide incidence and prevalence of $C$. trachomatis infections. To this end, various studies exploring the potential use of molecules with immune therapeutic properties $[19,26,27]$ are generating increasing interest, particularly for developing new methods to curtail the devastating consequences of the inflammatory aspect of the disease.

Interleukin-10 (IL-10) is a molecule with potent antiinflammatory therapeutic properties [28-30]. It is a multifunctional immuno-regulatory cytokine that plays a central role in suppressing inflammation, preventing damage to the host, and maintaining normal tissue homeostasis [31, 32]. IL-10 has significant effects on immune cells, specifically related to antigen presentation, the release of immune mediators, and phagocytosis [32, 33]. The immunosuppressive activity of IL-10 is mediated by its heterodimeric IL-10 receptor (IL-10R1, IL-10R2), whose ligation activates the Janus kinases (JAKs), signal transducer and activator of transcription proteins (STATs) (JAK/STAT) signaling and subsequently leading to massive changes in the expression profile of immuno-modulatory genes [34]. These genes effectively serve to enhance the IL-10 inhibitory, tolerance, and scavenger functions of monocytes and macrophages [35].

IL-10 and other cytokines that are involved in the regulation of the immune system and inflammation [36] use the JAK/STAT pathway, which in turn is regulated, especially by the suppressors of cytokine signaling (SOCS) proteins [34]. SOCS proteins fine-tune immune responses by binding to JAK and other cytokine receptors to suppress signaling events, thereby serving as key physiological regulators of inflammation [37, 38]. Reportedly, SOCS1 and SOCS3 are essential regulators of adaptive immunity, making them ideal therapeutic targets for inflammatory diseases such as Chlamydia. More importantly, IL-10 induces the expression of SOCS1 and SOCS3 in macrophages, suggesting that SOCS proteins may be mediators of its anti-inflammatory actions [39].

We previously reported that infection of mouse J774 macrophages with live $C$. trachomatis induces the release of IL-6, TNF, and IL-8, which were inhibited by IL-10 [27]. However, the chlamydial protein moiety responsible for inducing these inflammatory mediators and the mechanism(s) by which IL-10 inhibits them in macrophages are still unknown. We hypothesized that the Chlamydia MOMP mediates its disease pathogenesis, and SOCS1 and SOCS3 proteins are mediators of the IL-10 modulatory actions in macrophages. Our hypothesis was tested by first exposing J774 macrophages to dose-dependent concentrations of chlamydial stimulants [live C. muridarim $(\mathrm{Cm})$ and its recombinant MOMP] with and without exogenously added IL-10 to decipher the primary inducer of inflammatory mediators that are inhibited by IL-10. Second, we evaluated the timeand dose-dependency effect of stimulants alone or combined with IL-10 on the mRNA gene transcripts and protein expression of SOCS1 and SOCS3. Third, specific inhibitions of mitogen-activated protein kinase (MAPK) pathways (p38, JNK, and MEK1/2) were conducted to elucidate their requirements for the induction of inflammatory mediators as well as SOCS1 and SOCS3. Fourth, the role of endogenously produced IL-10 in regulating inflammatory mediators, macrophage phenotypes, and SOCS1 and SOCS3 expressions was investigated. Fifth, we examined the effect of IL-10 polarization on M1 and M2 macrophage phenotypes and the expression of SOCS1 and SOCS3 for its modulatory actions. Lastly, proteasome inhibition was explored to ascribe a functional role for SOCS1 and SOCS3 as mediators of the IL-10 anti-inflammatory effect in macrophages. We present our findings and discuss the multifaceted mechanisms by which IL-10 controls chlamydial inflammatory responses in macrophages.

\section{Materials and Methods}

2.1. Cell Line. Mouse J774 macrophages were obtained from the American Type Culture Collection (ATCC, Manassas, VA, USA) and cultured in Dulbecco Modified Eagle Medium (DMEM) (ATCC) supplemented with 10\% heat-inactivated fetal bovine serum (FBS) (Gibco, Grand Island, NY, USA) and $1 \mu \mathrm{g} / \mathrm{mL}$ antibiotic and antimycotic (Gibco) complete 
medium [40]. Cells were maintained at $37^{\circ} \mathrm{C}$ in a humidified incubator containing $5 \% \mathrm{CO}_{2}$ for various time-periods, depending on the experimental procedure.

2.2. Chlamydia Stimulants. Cm [strain Nigg II; previously called C. trachomatis mouse pneumonitis (MoPn) biovar] expressed as inclusion forming units (IFU/mL) was purchased from Virusys Corporation (Taneytown, MD, USA) [41]. The purified live Cm elementary bodies (EBs) were suspended in Sucrose-Phosphate Glutamic acid (SPG) buffer and stored in small aliquots at $-80^{\circ} \mathrm{C}$ until used. $\mathrm{Cm}$ was incubated at a multiplicity of infection (MOI) of $0.5,1$, and 2 with macrophages in antibiotic-free DMEM supplemented with $10 \%$ FBS. The recombinant major outer membrane protein (rMOMP) was cloned, as previously reported [11] and incubated with macrophages in complete media at concentrations ranging from $0.1,1$, and $10 \mu \mathrm{g} / \mathrm{mL}$.

2.3. Stimulation of Macrophages. Several experimental studies were considered to determine the effect of mouse recombinant IL-10 (BD Biosciences, San Jose, CA, USA) and chlamydial stimulants on the expression of cytokines and chemokines as well as SOCS1 and SOCS3.

Macrophages $\left(1 \times 10^{6} / \mathrm{mL}\right)$ were incubated in 12 -well plates and exposed to dose-dependent additions of rMOMP $(0.1,1$ and $10 \mu \mathrm{g} / \mathrm{mL})$ and $\mathrm{Cm}$ (MOI of $0.5,1$ and 2$)$ in the presence or absence of IL-10 $(10 \mathrm{ng} / \mathrm{mL})$ for $24 \mathrm{~h}$. For the IL-10-dose-dependent study, macrophages $\left(1 \times 10^{6} / \mathrm{mL}\right)$ were incubated in 12 -well plates with various concentrations of IL-10 $(0.1,1$ and $10 \mathrm{ng} / \mathrm{mL})$ in the presence or absence of rMOMP $(10 \mu \mathrm{g} / \mathrm{mL})$ and Cm (MOI of 2$)$ for $24 \mathrm{~h}$. Timekinetics studies were conducted by incubating macrophages $\left(1 \times 10^{6} / \mathrm{mL}\right)$ in 24 -well plates with various concentrations of IL-10 $(0.1,1$ and $10 \mathrm{ng} / \mathrm{mL})$ in the presence or absence of rMOMP $(10 \mu \mathrm{g} / \mathrm{mL})$ for $0.5,1,2$ and $24 \mathrm{~h}$ post-stimulation.

Pathway inhibition studies were performed using pharmacological inhibitors for p38 MAPK, JNK, and MEK1/2 signaling pathways. Macrophages $\left(1 \times 10^{6}\right.$ cells $\left./ \mathrm{mL}\right)$ were pre-incubated with $20 \mu \mathrm{M}$ pathway-specific inhibitor: SB203350 (p38 MAPK), SP600125 (JNK) and U0126 (MEK1/2) all from EMD Millipore Corporation (Billerica, MA, USA). After $1 \mathrm{~h}$ of pre-incubation, cells were exposed to $\operatorname{rMOMP}(10 \mu \mathrm{g} / \mathrm{mL})$ in the presence and absence of IL-10 $(10 \mathrm{ng} / \mathrm{mL})$ for an additional $24 \mathrm{~h}$. The $20 \mu \mathrm{M}$ concentration and $24 \mathrm{~h}$ inhibition time-point used for all inhibitors were optimal conditions as predetermined in our laboratory [19].

For the exogenous study, recombinant IL-10, IL-6, and TNF (BD Biosciences) each at $10 \mathrm{ng} / \mathrm{mL}$ was added to macrophages in the presence and absence of rMOMP $(10 \mu \mathrm{g} / \mathrm{mL})$ and $\mathrm{Cm}$ (MOI of 2). For the endogenous study, neutralization of endogenously produced IL-10, IL-6, and TNF was performed by pre-incubating macrophages with a neutralizing rat anti-mouse IL-10 antibody (Ab), anti-mouse IL-6 $\mathrm{Ab}$ and anti-mouse TNF $\mathrm{Ab}$ (each at $25 \mu \mathrm{g} / \mathrm{mL}$ ) Normal rat IgG1 $\mathrm{Ab}(25 \mu \mathrm{g} / \mathrm{mL})$ served as the isotype control. After $30 \mathrm{~min}$ of pre-incubation at $37^{\circ} \mathrm{C}$, cells were exposed to $\operatorname{rMOMP}(10 \mu \mathrm{g} / \mathrm{mL})$ or $\mathrm{Cm}$ (MOI of 2$)$ for an additional $24 \mathrm{~h}$.
M1 and M2 macrophage phenotypes were determined using stimulated macrophages from the dose-dependent and neutralization studies above. For M1 and M2 polarization studies, macrophages were pre-incubated with Interferongamma (IFN- $\gamma$ ), Interleukin-4 (IL-4), Interleukin-13 (IL-13) or IL-10 (each at $10 \mathrm{ng} / \mathrm{mL}$ ) for $1 \mathrm{~h}$ before stimulation with rMOMP $(10 \mu \mathrm{g} / \mathrm{mL})$ in the presence and absence of IL-10 $(10 \mathrm{ng} / \mathrm{mL})$ for an additional $24 \mathrm{~h}$.

For inhibition of proteasome degradation, macrophages $\left(1 \times 10^{6} / \mathrm{mL}\right)$ were pre-treated with the FDA approved proteasomal inhibitor; Bortezomib (Btzb) (Millipore-Sigma Aldrich, St. Louis, MO, USA) at concentrations of 1 and $20 \mathrm{nM}$ for $1 \mathrm{~h}$, followed by stimulation with rMOMP $(1 \mu \mathrm{g} / \mathrm{mL})$ and $\mathrm{Cm}$ (MOI of 2$)$ with or without added IL-10 $(10 \mathrm{ng} / \mathrm{mL})$ for an additional $24 \mathrm{~h}$.

All stimulated macrophage cultures were incubated at $37^{\circ} \mathrm{C}$ under $5 \% \mathrm{CO}_{2}$ for various time-points ranging between $30 \mathrm{~min}$ to $24 \mathrm{~h}$ depending on the specific experiment. Poststimulation, cell-free supernatants were collected by centrifugation at $450 \times \mathrm{g}$ for $10 \mathrm{~min}$ at $4^{\circ} \mathrm{C}$ and stored at $-80^{\circ} \mathrm{C}$ until used for cytokine and chemokine ELISAs. Cell pellets were either used for RNA extraction or flow cytometry analysis, as described below.

2.4. Quantification of Cytokines and Chemokines. For all studies, cytokines and chemokines were quantified in cellfree supernatants using cytokine and chemokine specific ELISAs as reported $[11,40]$. Cytokine kits (IL-6, IL-10, IL-12 p40, TNF) were purchased from BD Biosciences and BioLegend (San Diego, CA, USA). Chemokine kits (CCL5 and CXCL10) were purchased from R\&D Systems (Minneapolis, MN, USA). Absorbance was read at $450 \mathrm{~nm}$ using a microplate reader (Hidex Chameleon, IN, USA). The detection limits were $4 \mathrm{pg} / \mathrm{mL}$ (IL-6, IL-10, IL-12p40 and TNF), $31 \mathrm{pg} / \mathrm{mL}$ (CCL5), and $62 \mathrm{pg} / \mathrm{mL}$ (CXCL10). All ELISAs were run in triplicates and repeated at least 4 times.

2.5. RNA Extraction and Quantitative Real Time-PCR ( $q R T$ $P C R)$. Total RNA was isolated from unstimulated and stimulated cells using Qiagen RNeasy mini plus Kit (Qiagen Inc., Valencia, CA, USA), which included a DNase-I digestion step or the use of gDNA eliminator columns. The resulting RNA samples were transcribed into complementary deoxyribonucleic acid (cDNA) using the High Capacity cDNA Reverse Transciption Kit (Applied Biosystems, Foster City, CA, USA). Next, TaqMan ${ }^{\circledR}$ qRT-PCR was employed as described [42] to assess the messenger ribonucleic acid (mRNA) gene transcripts of the following genes (socs1 [Mm00782550_s1], socs3 [Mm00545913_ s1], stat1 [Mm01219775_m1], stat3 [Mm01219775_m1], macrophage mannose receptor (mrc1) [Mm01329362_ $\mathrm{m} 1$ ], arginase 1 (arg1) [Mm00475988_m1] and nitric oxide synthase 2 (nos2) [Mm00440502_m1]) using TaqMan ${ }^{\circledR}$ gene expression assays (Applied Biosystems) as reported [19, 26, 41]. Amplification of gene transcripts was performed according to the manufacturer's protocol using ABI ViiA ${ }^{\mathrm{TM}} 7$ realtime PCR (Applied Biosystems) and standard amplification conditions. The relative changes in gene expression were calculated using the equation: ${ }^{2-} \Delta \Delta \mathrm{CT}$ where all values were 
normalized with respect to the "housekeeping" gene glyceraldehyde 3-phosphate dehydrogenase (GAPDH) [Mm99999915_ g1] mRNA levels. Amplification using $50 \mathrm{ng}$ RNA was performed in a total volume of $20 \mu \mathrm{L}$. Each real-time PCR assay was performed in triplicates and repeated at least 4 times.

2.6. Immunofluorescence Microscopy. Macrophages $\left(2.5 \times 10^{4}\right.$ cells/well) were cultured on sterilized 8-well chamber slides and exposed to $\mathrm{Cm}$ (MOI of 1$)$ or $\operatorname{rMOMP}(1 \mu \mathrm{g} / \mathrm{mL})$ in the presence or absence of IL-10 $(10 \mathrm{ng} / \mathrm{mL})$ as described [27]. After $24 \mathrm{~h}$ post-exposure, the supernatants were removed; the cells were washed with phosphate-buffered saline (PBS), fixed with 2\% paraformaldehyde (PFA), and then permeabilized in permeabilization buffer $(\mathrm{PB})$ containing Saponin $(0.5 \%)$ for $0.5 \mathrm{~h}$. Permeabilized cells were subsequently subjected to immunostaining using fluorochromeconjugated anti-SOCS3 Alexa Fluor ${ }^{\circledR} 647$ primary antibody (Santa Cruz Biotechnology, Dallas, TX, USA) diluted in PB. After $1 \mathrm{~h}$ incubation at room temperature (RT), the cells were washed with PBS then stained with DAPI and mounted using Vectashield $^{\circledR}$ Hardset $^{\mathrm{TM}}$ anti-fade mounting medium with Phalloidin (Vector Laboratories, Burlingame, CA, USA). The slides were then visualized under an epifluorescence microscope equipped with Digital sight DS-Qil HighDefinition camera and NIS-Elements AR software (Nikon Instrument, Melville, NY, USA).

2.7. Flow Cytometry. Macrophages were stimulated (section 2.3 above), washed and blocked with Fc blocking Ab (BD Biosciences) in fluorescent-activated cell sorting (FACS) buffer (PBS, $0.1 \% \mathrm{NaN}_{3}, 1.0 \%$ FBS for $15 \mathrm{~min}$ at $4^{\circ} \mathrm{C}[11,12]$. The cells were washed and stained with fluorochrome-conjugated antibodies (Abs) (SOCS1-Alexa Fluor ${ }^{\circledR}$ 488, SOCS3-Alexa Fluor $^{\circledR}$ 647, NOS2-PE and MRC1/CD206-Alexa Fluor ${ }^{\circledR} 680$ (Santa Cruz Biotechnology, Dallas, TX, USA)) for $30 \mathrm{~min}$ at $4^{\circ} \mathrm{C}$, and then washed, fixed with $2 \%$ paraformaldehyde solution (PFA) for $20 \mathrm{~min}$ at $4^{\circ} \mathrm{C}$. Data were acquired on a $\mathrm{BD}$ FACS Canto II flow cytometer (BD Bioscience) with at least $1 \times 10^{5}$ events for each sample and analyzed using FCS Express 6 FLOW (De Novo Software, Pasadena, CA, USA).

2.8. Statistics Analysis. Data are expressed as the mean \pm standard deviation (SD) of samples run in triplicates, and each experiment was repeated at least 3 to 4 different times. Statistical analyses were performed using one- or two-way analysis of variance (ANOVA) followed by Tukey's Post-test using GraphPad Prism 6 Software (GraphPad Software, Inc., San Diego, CA, USA). Statistical significance was established and $\mathrm{P}$ values $<0.05$ were considered as statistically significant $(* P<0.05 ; * * P<0.01, * * * P<0.001$ and $* * * * P<0.0001)$.

\section{Results}

3.1. Chlamydia MOMP Induces Copious Levels of Cytokines and Chemokines That Are Dose-Dependently Inhibited by Exogenous IL-10 in Macrophages. The secretion of inflammatory cytokines and chemokines plays an integral role in the pathogenesis of chlamydial diseases. We previously established that Chlamydia induces the secretion of several inflammatory cytokines and chemokines by mouse macrophages $[19,26,27]$ and that IL-10 effectively inhibited the production of TNF, IL-6, and IL-8 as elicited by Chlamydia in mouse macrophages [27]. Because our previous study employed live Chlamydia to test the inhibitory effect of IL10 on secreted cytokines, and knowingly that live bacteria are composed of a plethora of stimulatory molecules, we deemed it necessary to identify a protein moiety that is the major stimulator of inflammatory responses in macrophages. To this end, MOMP was selected because it is the most dominant and immunogenic surface protein on Chlamydia [43-46]. Macrophages were exposed to $\operatorname{rMOMP}(0.1,1$ and $10 \mu \mathrm{g} / \mathrm{mL}$ ) or $\mathrm{Cm}$ (MOIs of $0.5,1$ and 2) with and without added IL-10 $(10 \mathrm{ng} / \mathrm{mL})$. All concentrations of rMOMP significantly $(P<0.001)$ elicited the production of cytokines and chemokines in a concentration-dependent manner, except IL-12p40. Of interest, IL-10 inhibited their release and was highly effective at lower stimulant concentrations, suggesting IL-10 inhibitory action is dependent on the concentration of mediators in the milieu (Figures 1(a)-1(d)). $\mathrm{Cm}$ stimulated several-fold less cytokines and chemokines, and the addition of IL-10 significantly $(P<0.001$ to 0.05$)$ reduced their levels (Figures $1(\mathrm{e})-1(\mathrm{~h})$ ). However, an MOI of 2 corresponds to approximately $0.2 \mu \mathrm{g} / \mathrm{mL}$, which may help explain the lower stimulatory potential of $\mathrm{Cm}$.

To assess whether the IL-10 anti-inflammatory effect is concentration-dependent, we evaluated a dose-dependent inhibitory effect of IL-10 on the expression of inflammatory mediators in chlamydial-stimulated macrophages. Neither IL-10 or unstimulated macrophages induced cytokines or chemokines. As expected, IL-10 at all tested concentrations $(0.1,1$, and $10 \mathrm{ng} / \mathrm{mL})$ significantly $(P<0.001$ to 0.0001$)$ inhibited cytokines and chemokines in a dose-dependent fashion, being more effective at the $10 \mathrm{ng} / \mathrm{mL}$ concentration (Figures 1(i)-1(p)). Notably, IL-10 was more efficient in inhibiting cytokines than chemokines. Our results confirm that MOMP is a significant inducer of inflammatory mediators in macrophages and that the IL-10 maximal inhibitory effect is dependent on the concentration of mediators in the milieu. Notably, the data also shed new light on the IL10-mediated inhibition of both chlamydial-induced cytokines and chemokines.

3.2. SOCS1 and SOCS3 Are Differentially Induced at the Transcriptional Level and Regulated by Exogenous IL-10 in Chlamydia-Stimulated Macrophages. It has been previously shown that SOCS1 and SOCS3 expressions can be induced in macrophages by IL-10 [39, 47-52], and their expressions are frequently increased in many inflammatory diseases $[37,53-56]$, such as Chlamydia $[57,58]$. Therefore, we aimed to decipher the molecular mechanism(s) by which IL-10 inhibits Chlamydia inflammatory responses in macrophages by focusing on SOCS1 and SOCS3. Both dose-dependent and time-kinetics experiments were performed using macrophages stimulated with rMOMP $(10 \mu \mathrm{g} / \mathrm{mL})$ in the presence and absence of IL-10 (0.1, 1 and $10 \mathrm{ng} / \mathrm{mL})$ for various time-points $(0.5,1,2$ and $24 \mathrm{~h})$. The time-kinetics and dose-dependent studies revealed that IL-10 marginally induced SOCS1 in contrast to SOCS3 mRNA gene 


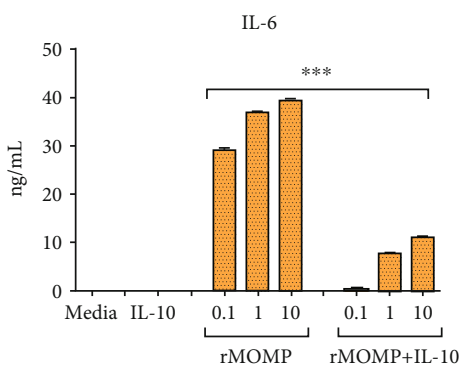

(a)

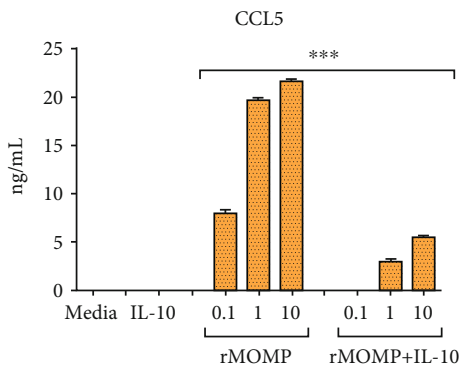

(c)

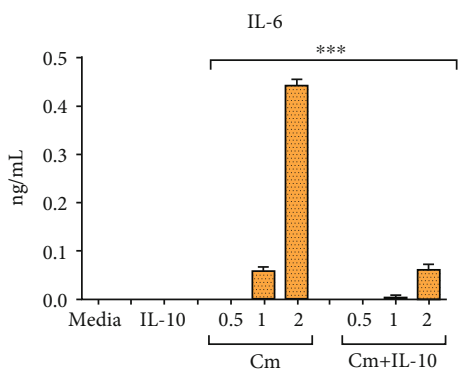

(e)

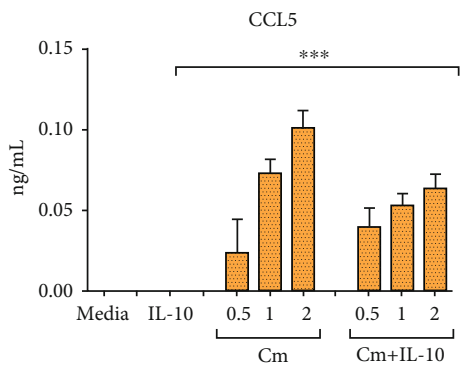

(g)

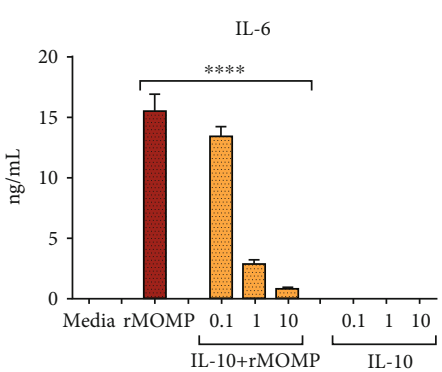

(i)

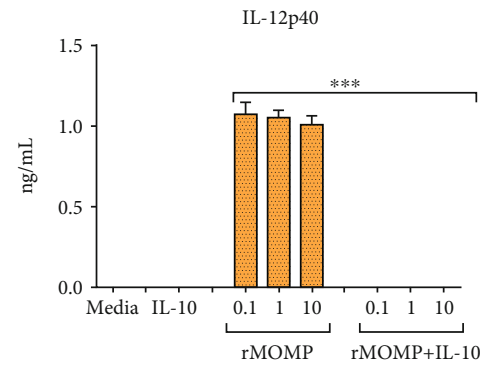

(b)

CXCL10

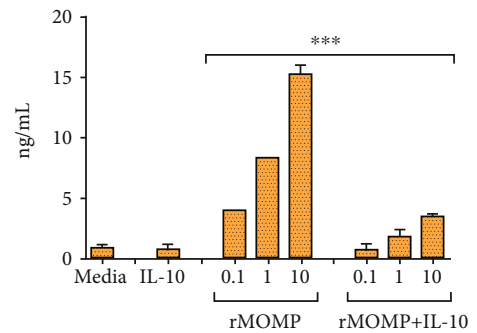

(d)

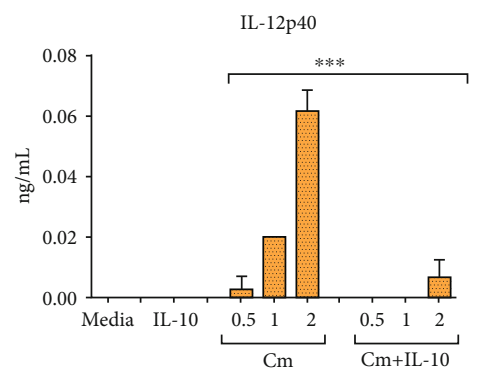

(f)

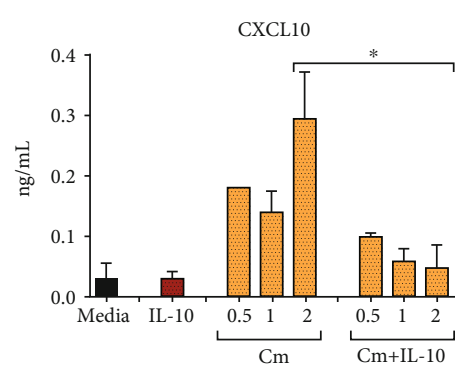

(h)

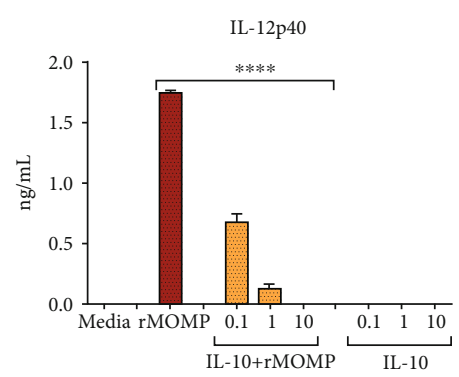

(j)

FIgURE 1: Continued. 


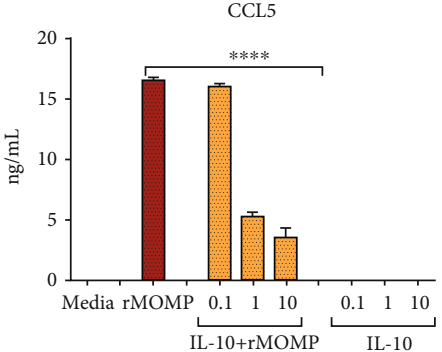

(k)

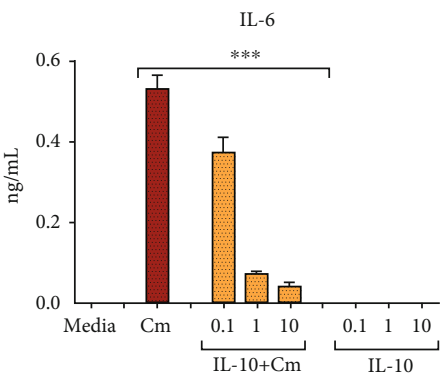

(m)

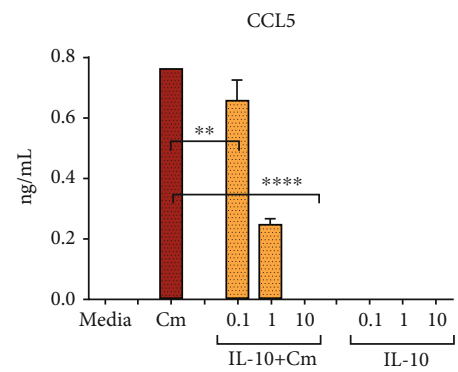

(o)

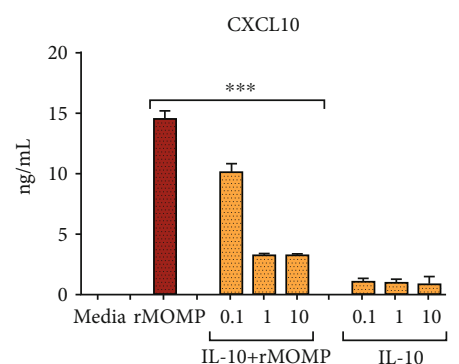

(l)

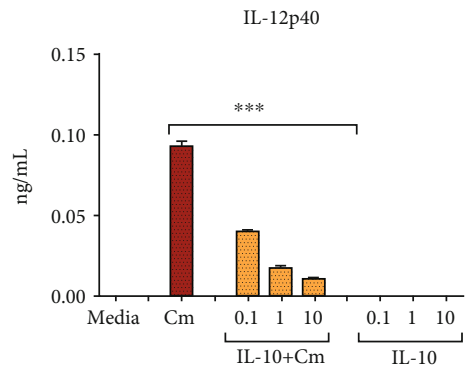

(n)

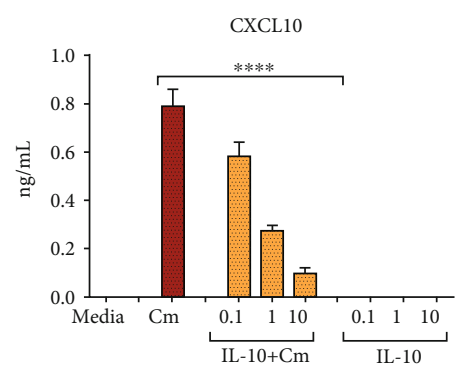

(p)

FIGURE 1: MOMP is a potent inducer of inflammatory mediators that are dose-dependently inhibited by added IL-10 to macrophages. For chlamydial stimulant dose-dependent studies, macrophages $\left(10^{6}\right.$ cells $\left./ \mathrm{mL}\right)$ were stimulated with $\mathrm{rMOMP}$ at $0.1,1$ and $10 \mu \mathrm{g} / \mathrm{mL}(\mathrm{A}-\mathrm{D})$ or live $\mathrm{Cm}$ at MOIs of $0.5,1$ and $2(\mathrm{E}-\mathrm{H})$ in the presence and absence of IL-10 $(10 \mathrm{ng} / \mathrm{mL})$. For IL-10 dose-dependent studies, macrophages were stimulated with rMOMP at $10 \mu \mathrm{g} / \mathrm{mL}$ (I-L) or Cm at an MOI of 2 (M-P) in the presence and absence of varying dosages of IL-10 $(0.1,1$ and $10 \mathrm{ng} / \mathrm{mL})$. Cell-free culture supernatants were collected after $24 \mathrm{~h}$ to quantify cytokines and chemokines by specific ELISAs. Asterisks indicate significant differences between stimulated macrophages alone and those with added IL-10 $(P<0.05)$. $P$ values were calculated by the use of ANOVA followed by Turkey's Post-test using GraphPad Prism 6 Software. Each bar represents the mean \pm SD of samples run in triplicates. Each experiment was repeated at least 4 times with similar results and shown is a representative experiment.

transcripts, particularly at the $10 \mathrm{ng} / \mathrm{mL}$ concentration (Figures 2(a) and 2(b)). IL-10 rapidly induced SOCS3 in macrophages as early as $0.5 \mathrm{~h}$, with a steady increase up to $24 \mathrm{~h}$. We observed that rMOMP induced marked SOCS3 in contrast to SOCS1 expression over the $24 \mathrm{~h}$ period, suggesting their differential stimulatory expressions. Co-incubation of IL-10 and rMOMP confirmed the differential regulation of SOCS1 and SOCS3 by IL- 10 as early as $0.5 \mathrm{~h}$ by suppressing SOCS1, while simultaneously increasing SOCS3 expression. $\mathrm{Cm}$ (MOI of 2) likewise induced the differential expression of SOCS1 and SOCS3, albeit less, and IL-10 dose-dependently regulated their expression, particularly SOCS3 (Figure 2(c)).

Next, experiments were conducted to ascertain whether SOCS1 and SOCS3 expressions are dose-dependently induced by chlamydial stimulants and if the observed differential regulation of SOCS by IL-10 is dependent on the stim- ulant concentration. We show in Figure 2(d) that rMOMP dose-dependently upregulated SOCS1 and SOCS3, with again a SOCS3 expression. With added IL-10, SOCS1 was reduced, and SOCS3 enhanced; likewise, IL-10 regulation of SOCS1 and SOCS3 was directly proportional to the stimulant concentration. Similarly, $\mathrm{Cm}$ at all MOIs stimulated SOCS1 and SOCS3 gene transcripts, especially SOCS3, and the addition of IL-10 to increasing MOIs of Cm did not alter SOCS1 but significantly $(P<0.001)$ upregulated SOCS3 expression (Figure 2(e)). These findings confirm the differential regulation of chlamydial-induced SOCS1 and SOCS3 by IL-10, irrespective of the stimulant concentration.

To ensure the IL-10 differential regulation of SOCS, we calculated their SOCS1 and SOCS3 ratios, which are good indicators of the magnitude of their expression. As indicated in Figure 2(f), IL-10 reduced the SOCS1/SOCS3 ratios in rMOMP- and Cm-stimulated macrophages by $\sim 4$ - and 7 - 


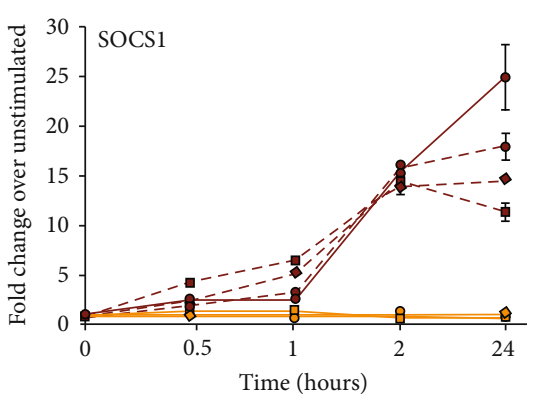

-- IL-10 $(0.1 \mathrm{ng} / \mathrm{mL})$

$\diamond$ IL-10 $(1 \mathrm{ng} / \mathrm{mL})$

- $\operatorname{IL}-10(10 \mathrm{ng} / \mathrm{mL})$

- $\operatorname{rMOMP}(10 \mu \mathrm{g} / \mathrm{mL})$

- rMOMP+IL-10 (0.1 ng/mL)

- rMOMP+IL-10 $(1 \mathrm{ng} / \mathrm{mL})$

- rMOMP+IL-10 $(10 \mathrm{ng} / \mathrm{mL})$

(a)

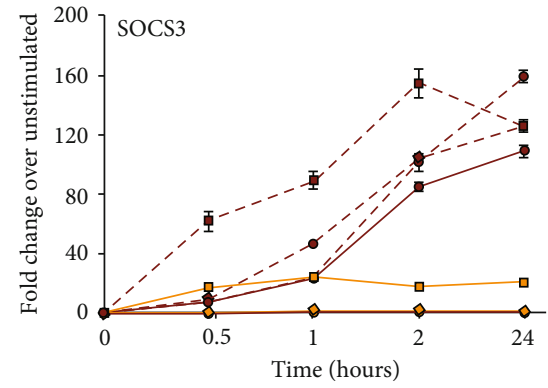

- IL-10 (0.1 ng/mL)

$\diamond$ IL-10 $(1 \mathrm{ng} / \mathrm{mL})$

—- IL-10 (10 ng/mL)

$\rightarrow \operatorname{rMOMP}(10 \mu \mathrm{g} / \mathrm{mL})$

- rMOMP+IL-10 $(0.1 \mathrm{ng} / \mathrm{mL})$

- rMOMP+IL-10 $(1 \mathrm{ng} / \mathrm{mL})$

๑- rMOMP+IL-10 (10 ng/mL)

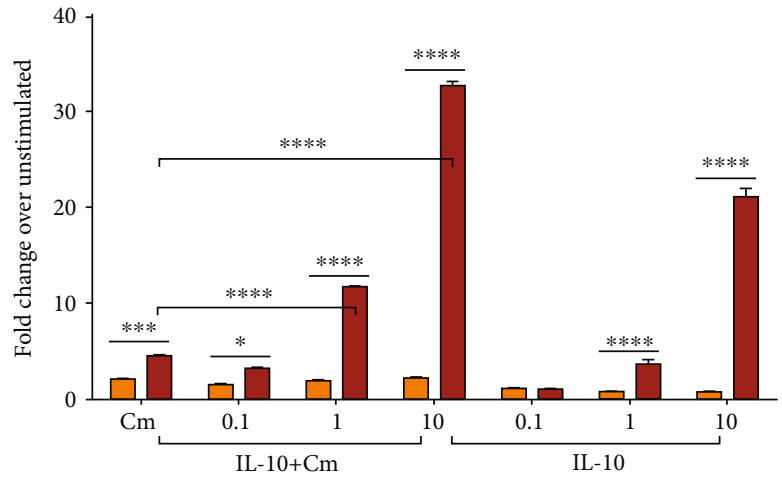

$\square$ SOCS1

$\square$ SOCS3

(c)

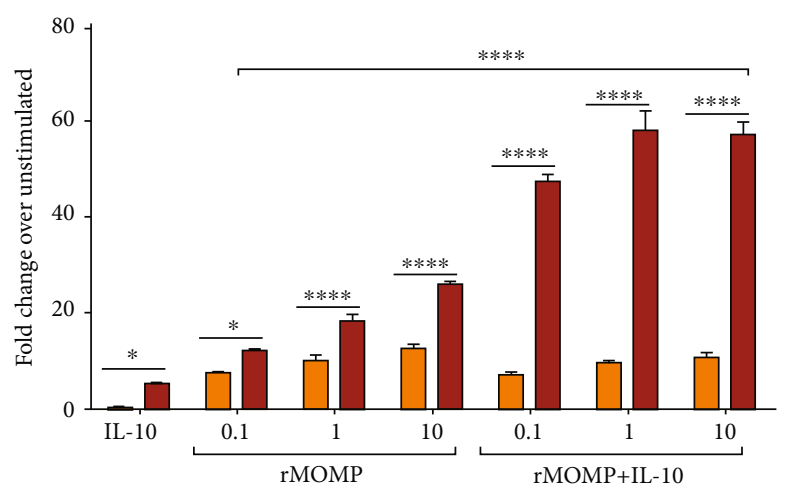

$\square$ SOCS1

SOCS3

(d)

FIgURe 2: Continued. 


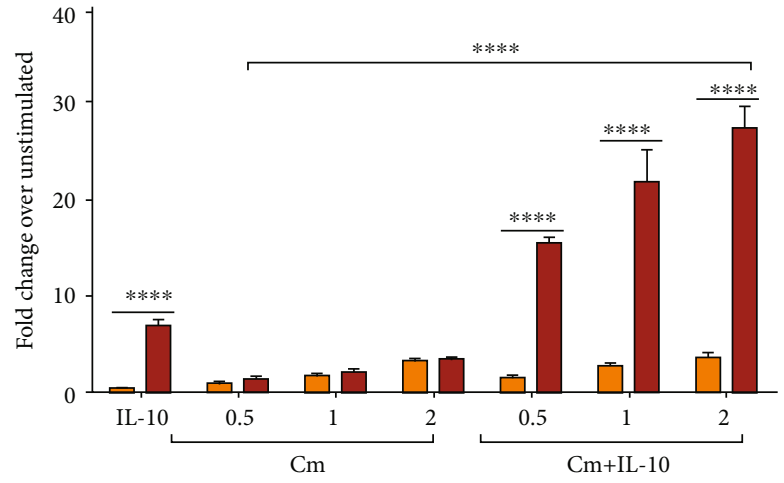

$\square$ SOCS1

$\square$ SOCS3

(e)

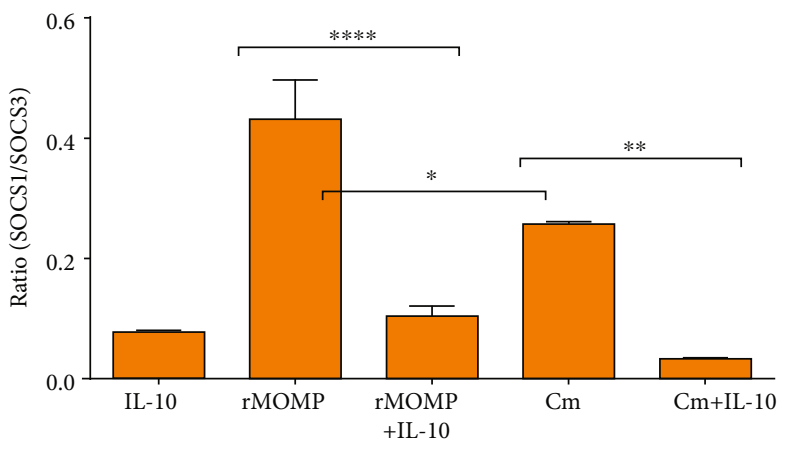

(f)

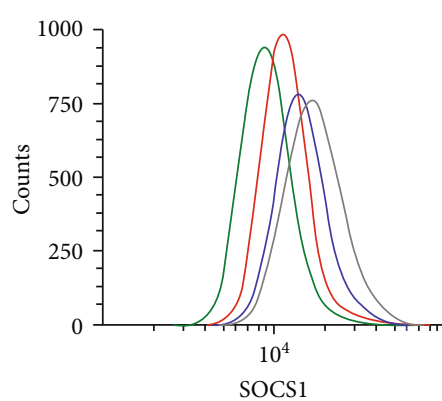

$\begin{array}{ll}\text { Cells } & \text { IL-10 } \\ \text { rMOMP } & \text { Cm }\end{array}$

(h)

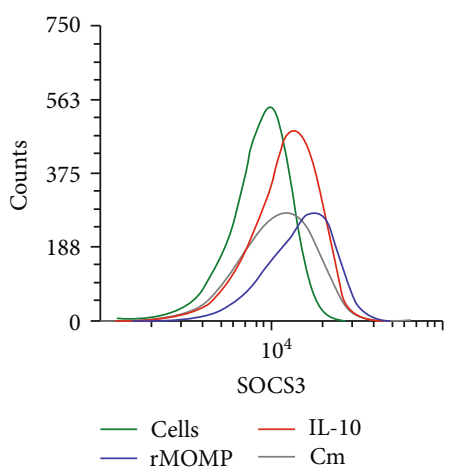

(k)

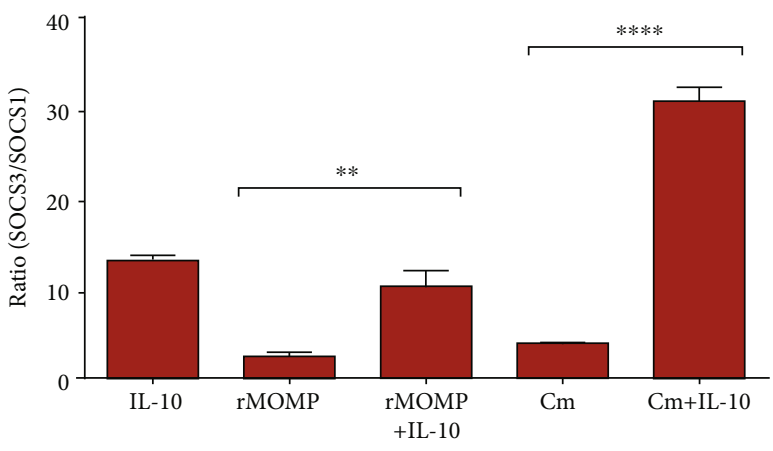

(g)

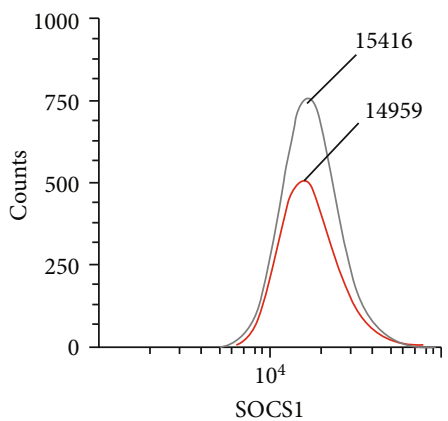

$\mathrm{Cm}$
$-\mathrm{Cm}+\mathrm{IL}-10$

(j)
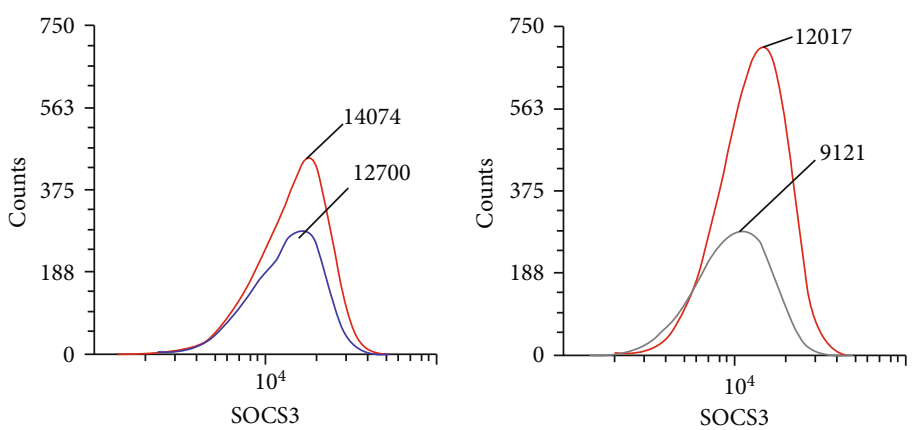

- rMOMP

- rMOMP+IL-10

(l)
$\mathrm{Cm}$
$\mathrm{C} \mathrm{Cm}+\mathrm{IL}-10$

(m)

Figure 2: Continued. 


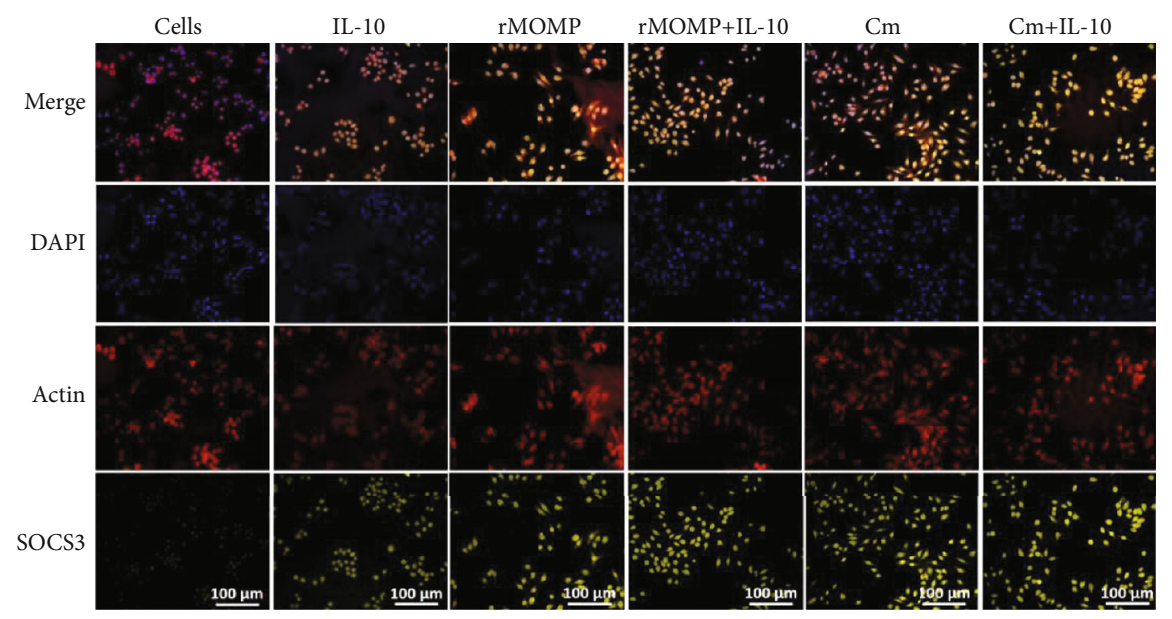

(n)

FIGURE 2: SOCS1 and SOCS3 transcriptional and translational expressions are differentially induced and regulated by chlamydial stimulants and exogenous IL-10 in macrophages. Macrophages were exposed to dose-dependent concentrations of IL-10 $(0.1,1 \mathrm{and} 10 \mathrm{ng} / \mathrm{mL}) \mathrm{in}$ the presence and absence of $\mathrm{rMOMP}(10 \mu \mathrm{g} / \mathrm{mL})$. RNA samples were collected at $0,0.5,1,2$, and $24 \mathrm{~h}$ post-stimulation to quantify the mRNA gene transcript of SOCS1 (A) and SOCS3 (B). RNA samples were collected at $24 \mathrm{~h}$ from macrophages stimulated with Cm (MOI of 2) with and without dose-dependent concentrations of IL-10 to quantify SOCS1 and SOCS3 transcripts (C). Macrophages were stimulated with $\operatorname{rMOMP}(0.1,1$ and $10 \mu \mathrm{g} / \mathrm{mL})$ or Cm (MOI of $0.5,1$ and 2$)$ with and without IL-10 (10 ng/mL) and SOCS1 and SOCS3 transcripts were quantified at $24 \mathrm{~h}$ post-stimulation (D-E). For TaqMan qRT-PCR, all values were normalized with respect to the mRNA levels of the "housekeeping" gene that codes for GAPDH. Results are presented as fold increase over the control (i.e., the level in unstimulated cells. Calculations of SOCS1/SOCS3 (F) and SOCS3/SOCS1 (G) ratios in macrophages exposed to rMOMP $(10 \mu \mathrm{g} / \mathrm{mL})$ or Cm (MOI of 2) in the presence and absence of IL-10 $(10 \mathrm{ng} / \mathrm{mL})$. Macrophages were exposed to rMOMP $(1 \mu \mathrm{g} / \mathrm{mL})$ or Cm (MOI of 2$)$ in the presence and absence of IL-10 to evaluate SOCS1 and SOCS3 expression by flow cytometry (H-M) and SOCS3 by immunofluorescence microscopy (N) $24 \mathrm{~h}$ post-incubation. An asterisk indicates significant differences $(P<0.05)$, and $P$ values were calculated as described in Figure 1 legend. Each bar represents the mean \pm SD of samples run in triplicates, and each experiment was repeated at least 4 times with a representative experiment shown.

fold, respectively, due to its down-regulation of SOCS1. Conversely, IL-10 enhanced, respectively, the SOCS3/SOCS1 ratio by $\sim 4$ - and 8 -fold fold in rMOMP and $\mathrm{Cm}$ cultures apparently by increasing SOCS3 expression (Figure 2(g)). These experiments infer that SOCS1 and SOCS3 are differentially induced and regulated by IL-10, and are key protagonists in the IL-10- inhibition of Chlamydia-induced inflammatory responses in macrophages.

The functions of SOCS1 and SOCS3 were quantified at the translational level employing flow cytometry to broaden our understanding of their expression and regulation as induced by chlamydial stimulants alone or with added IL10. Flow cytometric analyses revealed the expression of SOCS1 (Figure 2(h)), and SOCS3 (Figure 2(k)) in chlamydial- and IL-10-stimulated macrophages by their increased fluorescence intensities as compared to uninfected macrophages. Once more, IL-10 reduced SOCS1 (Figures 2(i) and 2(j)) and enhanced SOCS3 (Figures 2(l) and 2(m)) expression. Immunofluorescence microscopy was also employed to provide visual evidence for the expression and regulation of SOCS by IL-10 in chlamydial macrophages as supporting protein data. Only SOCS3 data is shown because of difficulties in attaining good images for SOCS1 due to their lower expression. In Figure 2(n), the rows, respectively, reflect overlay images (merge; bright yellow fluorescence), nuclei (DAPI; blue fluorescence), macrophage surface (actin; red fluorescence), and SOCS3 (yellow fluorescence). Our results demonstrate that unstimulated cells displayed negligible
SOCS3 expression. Although IL-10 upregulated SOCS3, it was lesser in contrast to rMOMP and $\mathrm{Cm}$ macrophages that exhibited increased bright yellow fluorescence aggregating around the nuclei, confirming their ability to induce high levels of SOCS3, which were enhanced with added IL-10. This study further confirms the induction of SOCS3 by chlamydial stimulants and the ability of IL-10 to regulate its translational expression and hence functions in macrophages.

\subsection{Effect of MAPK Pathway-Specific Inhibition on} Inflammatory Mediators and SOCS1 and SOCS3 Expressions. We investigated the downstream signaling pathways that may be involved in the induction and regulation of SOCS1 and SOCS3 in a Chlamydia-activating macrophage environment that may contain IL-10. We focused on MAPK pathways, specifically $\mathrm{p} 38, \mathrm{JNK}$, and $\mathrm{MEK} 1 / 2$, that reportedly play pivotal roles in regulating immune-mediated inflammatory responses [59-61] and SOCS functions [62]. Macrophages were pre-incubated for $1 \mathrm{~h}$ with pathway-specific inhibitors targeting p38 (SB203580), JNK (SP600125) and MEK1/2 (U0126) before stimulation with rMOMP with and without added IL-10. Macrophages exposed to IL-10 secreted minimal or no IL-6 or CCL5 (Figures 3(a) and 3(b)). We show in Figure 3(a) that the p38 inhibitor significantly repressed $(P<0.0001)$ IL-6 release, suggesting that p38 is necessary for IL-6 synthesis, corroborating reports that p38 inhibition reduces pro-inflammatory cytokine production [59, 63-66]. Inhibitors of the MEK $1 / 2$ and JNK pathways did not 


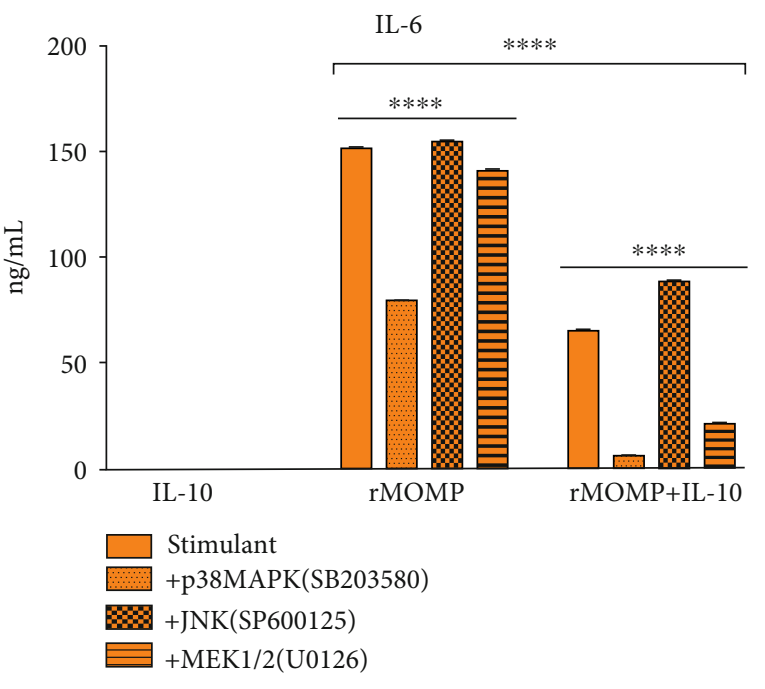

(a)

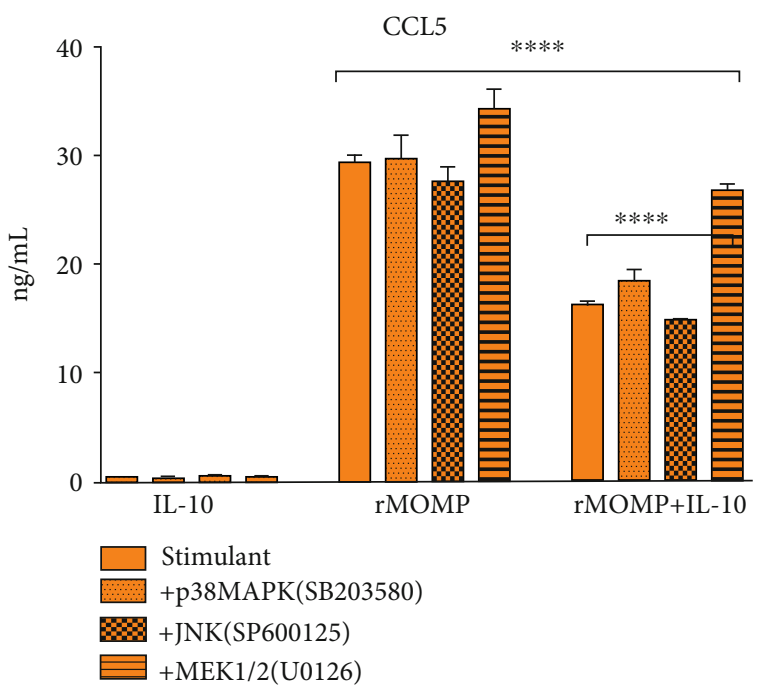

(b)

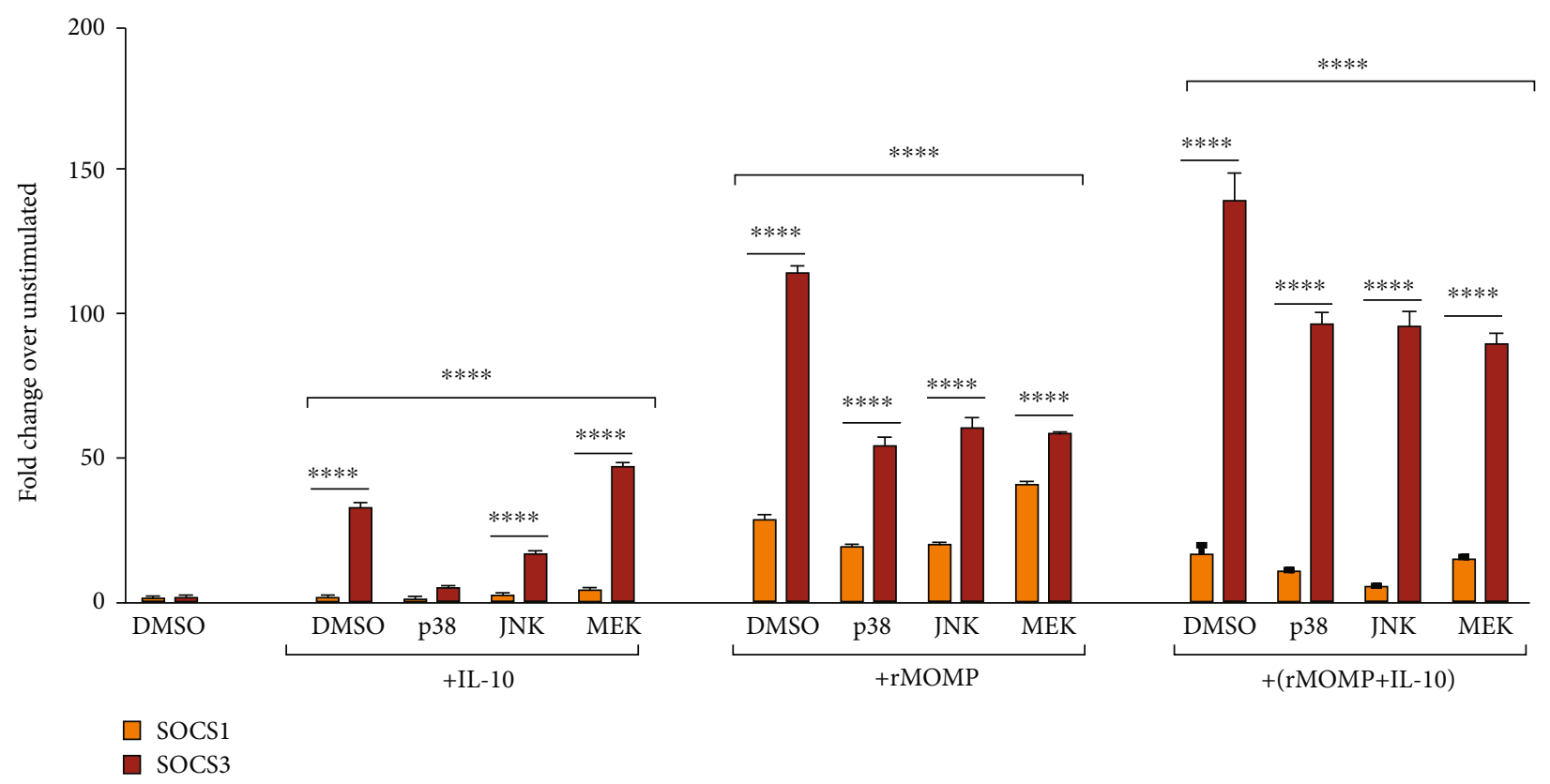

(c)

FIGURE 3: Specific MAPK pathways regulate the IL-10-inhibition of inflammatory mediators in chlamydial macrophages and the induction of SOCS expression. Macrophages $\left(10^{6} / \mathrm{mL}\right)$ were pre-incubated for $1 \mathrm{~h}$ with MAPK pathway specific-inhibitors (each at $\left.20 \mu \mathrm{M}\right)$ for $\mathrm{p} 38$ (SB203580), JNK (SP600125) and MEK1/2 (UO126) followed by stimulation for $24 \mathrm{~h}$ with $\mathrm{rMOMP}(10 \mu \mathrm{g} / \mathrm{mL})$ in the presence and absence of IL-10 (10 ng/mL). IL-6 (A) and CCL5 (B) were measured by specific ELISAs. TaqMan qRT-PCR was employed to quantify SOCS1 and SOCS3 mRNA gene transcripts (C). Results are presented as fold increase over the control. An asterisk indicates significant differences $(P<0.05)$, and $P$ values were calculated as described in Figure 1 legend. Each bar represents the mean \pm SD of samples run in triplicates. Each experiment was repeated 3 times.

appreciably alter IL-6 expression and, as such, not required for its synthesis. Both p38 and MEK1/2 inhibitors enhanced the IL-10 anti-inflammatory effect, implying that they regulate the magnitude of IL-10 inhibition of inflammatory mediators. Contrastingly, the JNK inhibitor enhanced IL-6, which reveals its necessity for IL-10 modulation of IL-6. Both p38 and JNK inhibitors did not alter CCL5 expression, but noticeably CCL5 release increased with MEK1/2 inhibition in IL-10 cocultures, suggesting that MEK1/2 blocks the IL-10 suppressive activity in macrophages (Figures 3(a) and 3(b)).
Next, quantification of SOCS1 and SOCS3 mRNA gene transcripts was conducted to determine the effect of pathway-specific inhibition on their expression, given the pivotal effects of specific pathways on cytokine and chemokine expression. Blockage of p38 caused the most notable decline in SOCS1 and SOCS3 mRNA expression, followed by JNK blockage, while blocking of MEK1/2 upregulated SOCS1 and SOCS3 expression as induced by IL-10. SOCS1 and SOCS3 transcripts were reduced by blocking p38 and JNK; conversely, MEK1/2 blockage enhanced SOCS1 
but reduced SOCS3. Of interest, inhibiting p38, JNK, and MEK1/2 in co-cultures of rMOMP and IL-10 significantly reduced $(P<0.001) \quad$ SOCS1 and SOCS3 expressions (Figure 3(a)). Collectively, these results reveal that MAPK pathways regulate the induction of SOCS1 and SOCS3 in macrophages as triggered by either IL-10 and rMOMP or a combination of both.

\subsection{Endogenous IL-10 Regulates Inflammatory Mediators and} SOCS Expression in Chlamydial-Stimulated Macrophages. Our data established that exogenous IL-10 effectively inhibited inflammatory mediators in macrophages exposed to chlamydial stimulants, and differentially regulated SOCS1 and SOCS3 expression. We questioned whether endogenously produced IL-10 might function similarly in chlamydial-stimulated macrophages. IL-10 secreted by rMOMP and $\mathrm{Cm}$ macrophages, respectively, were $381 \mathrm{pg} / \mathrm{mL}$ and $72 \mathrm{pg} / \mathrm{mL}$ (Figure 4(a)). Negative controls (isotype (ISO), anti-IL-10, and IL-10) did not induce any inflammatory mediators (Figures 4(b)$4(\mathrm{~g})$ ). Also, the anti-IL-10 Ab sufficiently neutralized the endogenously produced IL-10 in stimulated cultures, thus confirming its neutralization efficiency (Data not shown). Once more, rMOMP stimulated high levels of IL-6, IL$12 \mathrm{p} 40$, TNF and CCL5 and neutralization of the endogenously produced IL-10 resulted in the upregulation of these mediators, implying that endogenous IL-10 represses their maximal expression levels. Moreover, neutralization of exogenously added IL-10 with the anti-IL-10 Ab abolished the inhibitory effect of IL-10 in stimulated macrophages by restoring IL-6, IL-12-p40, TNF and CCL5 to comparable levels as induced by stimulants alone (Figures 4(b)-4(e)). These results were confirmed in $\mathrm{Cm}$-exposed cultures whereby IL- 6 and CCL5 were upregulated by neutralization of IL-10 (Figures 4(f)-4(g)). These findings reiterate that IL-10 (endogenous and exogenous) regulates the production of inflammatory mediators, thus solidifying the role of IL-10 in modulating inflammatory responses triggered by Chlamydia in macrophages.

To assess whether SOCS1 and SOCS3 mRNA gene transcripts would be altered by the removal of the endogenously produced IL-10, we quantified their expressions in chlamydial-stimulated macrophages. The removal of the endogenous IL-10 suppressed SOCS3 in both chlamydial cultures, proving that SOCS3 regulates the inflammatory responses. Co-culturing of the anti-IL-10 Ab with exogenous IL-10 lowered SOCS3 but not SOCS1 expression; thus confirming that exogenous and endogenous IL-10 effectively regulate SOCS expression (Figures 4(h)-4(i)). The reduced SOCS3 expression and its correlation with an increase in cytokines and chemokines support SOCS3 as a mediator of IL-10inhibitory activity in a chlamydial macrophage environment and underscores SOCS3 in mediating the inhibitory effects of IL-10 and its IL-10-mediated immune responses [49, 67].

3.5. Effect of Exogenous IL-6 and TNF on Inducing and Regulating Inflammatory Mediators as Well as SOCS1 and SOCS3 in Chlamydial-Stimulated Macrophages. The endogenous study solidified the anti-inflammatory role of IL-10 and with SOCS1 and SOCS3 as the putative mediators of its inhibitory actions in chlamydial macrophage cultures. Because chlamydial stimulants can induce a plethora of inflammatory cytokines, we questioned whether other cytokines might contribute to SOCS induction in macrophages. We targeted IL- 6 and TNF because they reportedly can stimulate SOCS1 and SOCS3 expression in macrophages [52]; and also to evaluate if they exhibit any anti-inflammatory properties. Macrophages were exposed to chlamydial stimulants with and without exogenously added IL-6, TNF and IL-10 to quantify IL-6 and CCL5. IL-10, as expected, decreased the expression of IL- 6 and CCL5; IL-6 reduced CCL5, and TNF did not alter IL-6 or CCL5 production levels in chlamydial-stimulated macrophages (Figures 5(a)-5(d)). This data shows that IL- 6 exhibits some anti-inflammatory effect, albeit to a lesser extent than IL-10.

We next investigated whether IL-6 and TNF contributed to SOCS1 and SOCS3 induction by chlamydial stimulants (Figures 5(e)-5(f)). Again, IL-10 alone or combined with stimulants significantly $(P<0.0001)$ upregulated SOCS3, while simultaneously decreasing SOCS1 expression. Exogenous IL-6 induced more SOCS3 than SOCS1 expression; conversely, their induction by TNF was weak. The addition of IL-6 to chlamydial macrophages significantly $(P<0.001$ to 0.0001 ) reduced only SOCS1 expression. Contrastingly, TNF enhanced SOCS1 and SOCS3 expressions only in Cm and not rMOMP cultures, suggesting possibly the triggering by other chlamydial antigens. These findings indicate that IL-6 but not TNF can induce SOCS3; however, both cytokines regulate SOCS1 and SOCS3 expression. We speculate that perhaps, to resolve and balance the addition of these pro-inflammatory mediators to the chlamydial milieu, IL-6 and TNF may display transient anti-inflammatory tendencies, consistent with their pleiotropic properties [68].

Neutralization of endogenously produced IL- 6 and TNF by their respective Abs did not significantly perturb rMOMP-induced SOCS1 and SOCS3 expression, implying their direct stimulation by rMOMP. Surprisingly, diminished SOCS1 and SOCS3 expressions occurred with blockade of endogenously produced IL- 6 and TNF in Cm-exposed cultures, which corroborate reports that SOCS1 and SOCS3 provide significant negative feedback interchangeably for IL-6 and TNF [69]. The removal of endogenously produced IL10 increased SOCS1 expression ( $\mathrm{Cm}$ only), and reduced SOCS3, suggesting an essential role of IL-10 in the regulation of SOCS1 and SOCS 3 expression (Figures 5(g)-5(h)). Collectively, our data sheds light on the potential roles of these cytokines implicated during chlamydial infections, but more importantly, how IL-10 regulates Chlamydia inflammation via SOCS proteins.

\subsection{Chlamydial Stimulants Trigger an M1 Pro-Inflammatory} Phenotype That Is Skewed to an M2 Anti-Inflammatory Phenotype in the Presence of Exogenous IL-10. We have established above that IL-10 upregulates SOCS3 while simultaneously downregulating SOCS1, suggesting its capacity to stimulate a therapeutic M2 macrophage phenotype. Therefore, we phenotypically characterized chlamydial-stimulated macrophages (M1 and M2) to address if IL-10 polarizes macrophage phenotypes to exert its anti-inflammatory actions 


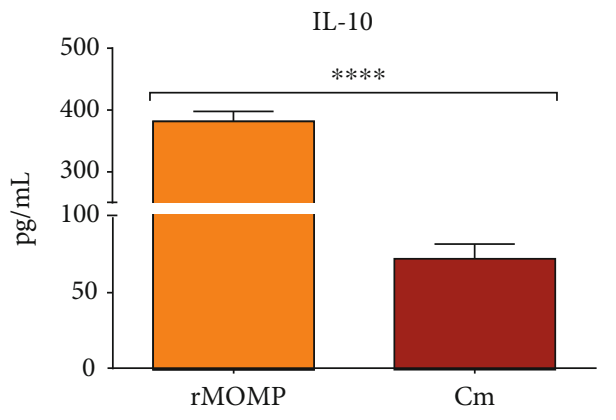

(a)

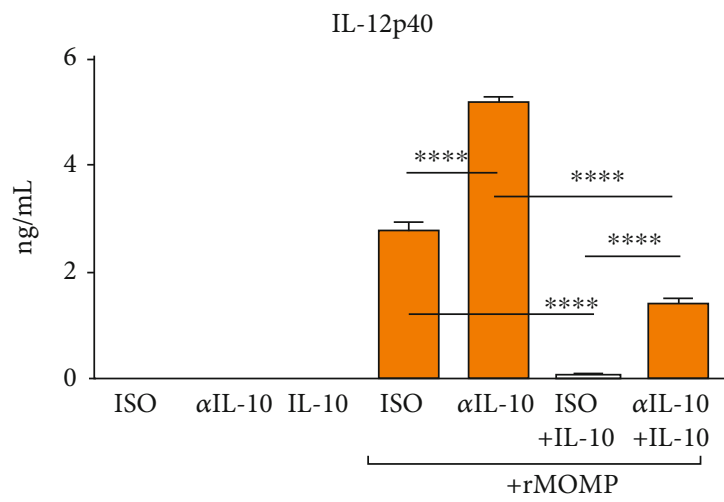

(c)

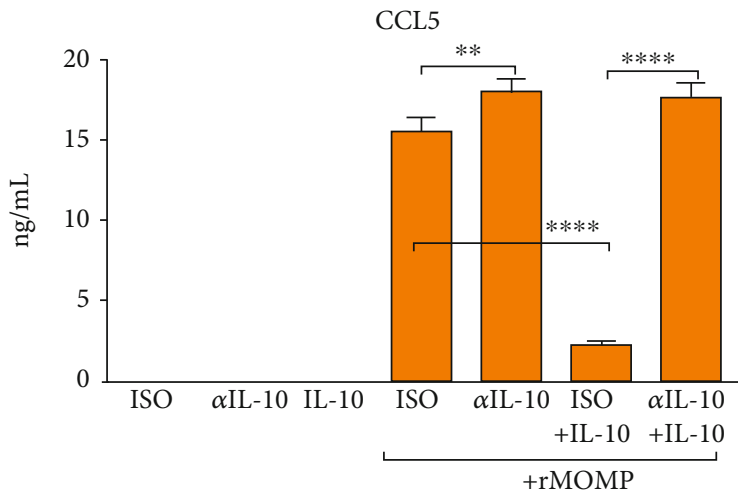

(e)

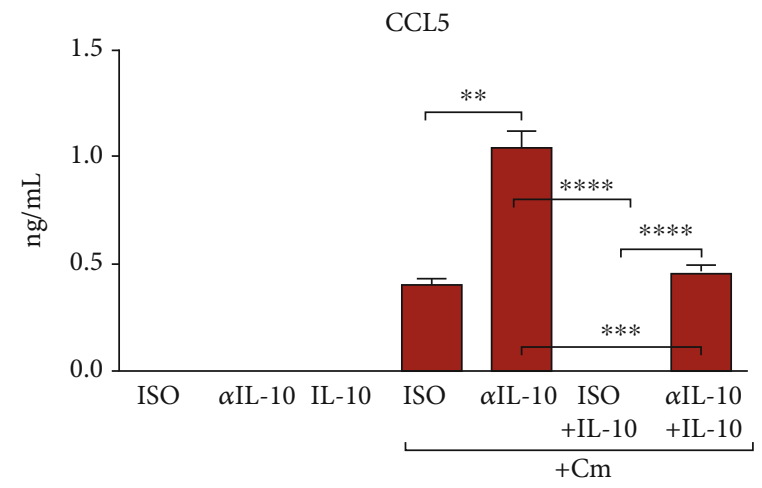

(g)
IL-6

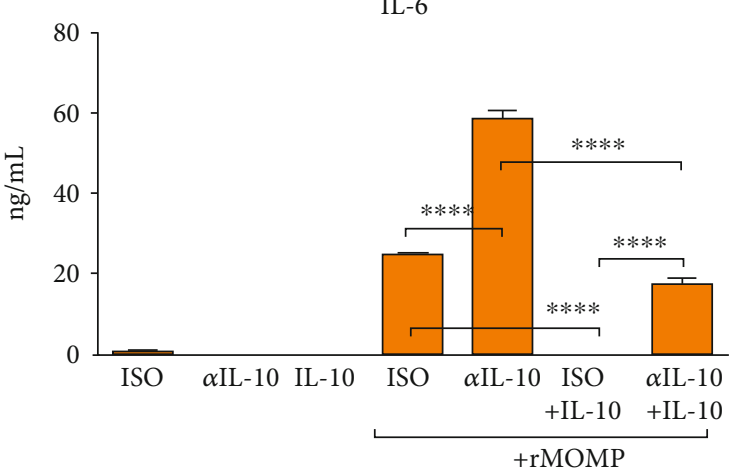

(b)

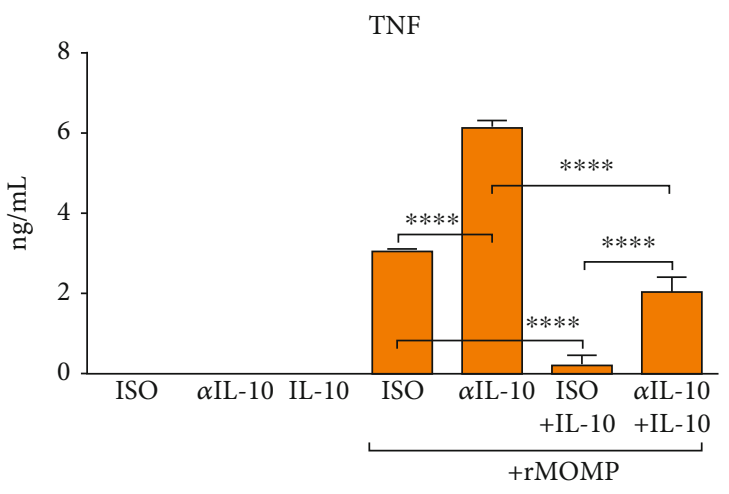

(d)

IL-6

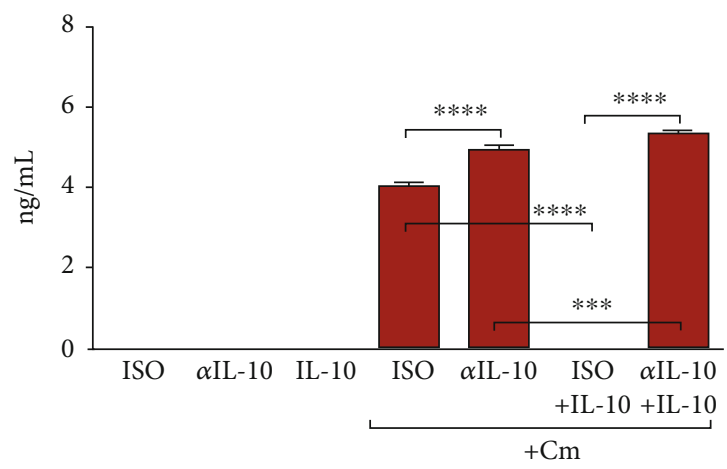

(f)

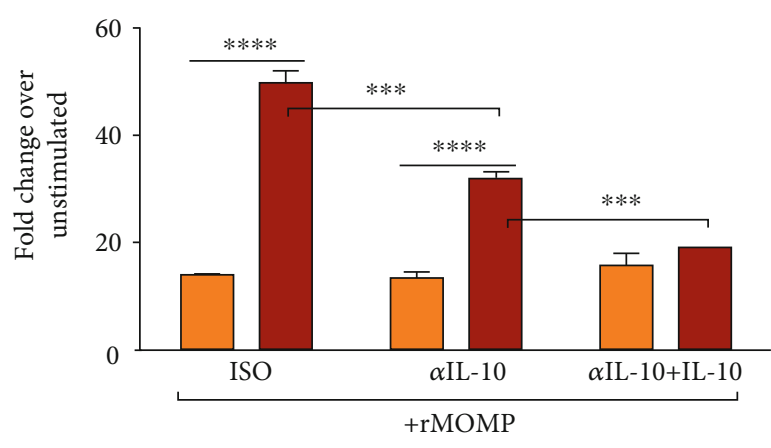

SOCS1

SOCS3
)
(h)

Figure 4: Continued. 


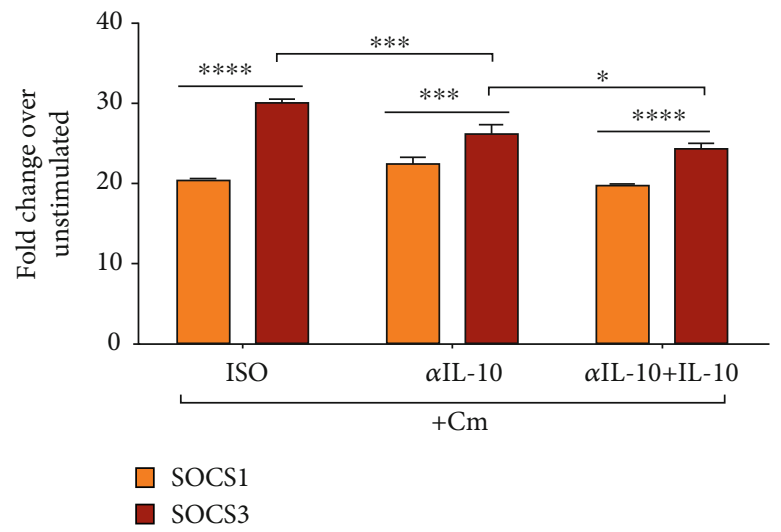

(i)

FIGURE 4: Endogenously produced IL-10 regulates inflammatory mediators and SOCS expression in chlamydial-stimulated macrophages. Macrophages $\left(10^{6} / \mathrm{mL}\right)$ were stimulated for $24 \mathrm{~h}$ with $\mathrm{rMOMP}(10 \mu \mathrm{g} / \mathrm{mL})$ or Cm (MOI of 2$)$ to quantify the production of endogenously produced IL-10 in supernatants by ELISA (A). Macrophages were pre-incubated with neutralizing Ab ( $\alpha \mathrm{IL}-10)$ to IL-10 (25 $\mu \mathrm{g} / \mathrm{mL})$ for $30 \mathrm{~min}$ before adding rMOMP $(10 \mu \mathrm{g} / \mathrm{mL})$ or Cm (MOI of 2$)$ for an additional $24 \mathrm{~h}$. Normal rat IgG1 Ab served as the isotype control (ISO). Post-stimulation, supernatants were collected to quantify IL-6 (B, F), IL-12p40 (C), TNF (D), and CCL5 (E, G) by specific ELISAs and RNA was isolated to quantify the mRNA gene transcripts of SOCS1 and SOCS3 (H-I) by TaqMan qRT-PCR. An asterisk indicates a significant difference $(P<0.05)$, and $P$ values were calculated as described in Figure 1 legend. Each bar represents the mean $\pm \mathrm{SD}$ of samples run in triplicates, and each experiment was repeated at least 3 times.

and to assess the role of SOCS1 and SOCS3 in skewing a chlamydial activating macrophage environment towards an M1 or M2 polarizing phenotype. Dose-dependent experiments were conducted using discriminate M1 (nos2) and M2 (arg1 and mrcl) markers to identify the macrophage phenotypic populations. Both M1 and M2 transcriptional expressions varied between experiments, but their expression patterns were consistent (Figures 6(a)-6(1)). Macrophages incubated with rMOMP or $\mathrm{Cm}$ at all tested concentrations expressed higher nos2 than those incubated with IL-10, implying the triggering of an M1 pro-inflammatory phenotype by chlamydial stimulants (Figures 6(a), 6(d), 6(g) and 6(j)). Interestingly, incubating IL-10 with stimulants significantly $(P<0.001)$ reduced nos 2 expression and skewing of the chlamydial M1 pro-inflammatory phenotype by IL-10. Nos2 expression increased with increasing concentrations of chlamydial stimulants, and IL-10 reduced nos2 expression at all tested dosages.

Our results for the M2 phenotypic markers conversely revealed that all concentrations of chlamydial stimulants induced minimal expression of mrcl and argl. In contrast, all dosages of IL-10 increased their expression, indicating selective stimulation of the M2 phenotype by IL-10 to possibly aid in mediating its anti-inflammatory actions (Figures 6(b)-6(c), 6(e)-6(f), 6(h)-6(i), 6(k)-6(l)). Coincubation of IL-10 with chlamydial stimulants enhanced the expression of mrcl and argl in macrophages, which infers that IL-10 skews chlamydial M1 towards an M2 phenotype to repress inflammation or that chlamydial stimulants skew M2 towards an M1 phenotype to balance macrophages polarizing functions in the microenvironment. We also calculated the M1 and M2 ratios as a predictive correlation of SOCS1 and SOCS3 expressions that regulate macrophage polarization. Depiction of the M1/M2 ratios indicates higher ratios for chlamydial stimulants, which are suggestive of enhanced M1 and SOCS3 expression, which were reduced with added IL-10 (Figure 6(m)). Contrastingly, higher M2/M1 ratios were attained for IL-10 and IL-10 coincubated with stimulants, indicative of an IL-10 polarizing M2 phenotype (Figure 6(n)).

Flow cytometric analysis similarly demonstrated at the protein level that chlamydial stimulants triggered the high expression of nos 2 with a concomitant lesser expression of mrc1. Once more, IL-10 reduced the expression of nos 2 while simultaneously increasing that of $\mathrm{mrcl}$, thus further illustrating the skewing of the chlamydial-induced M1 proinflammatory to an M2 anti-inflammatory phenotype at the translational level (Figures 6(o)-6(t)).

The observed skewing of the chlamydial M1 proinflammatory phenotype to an M2 anti-inflammatory macrophage phenotype by exogenous IL-10 begged to address the role of endogenously produced IL-10 in this phenomenon. As anticipated, IL-10 did not induce nos2 but rather upregulated mrcl (Figures 6(u)-6(x)). Chlamydial stimulants in the presence of the isotype control Ab (ISO) upregulated nos2. But neutralization of the endogenously produced IL-10 significantly upregulated $(P<0.0001)$ nos 2 , suggesting that the endogenously produced IL-10 regulates the M1 phenotype comparable to the exogenous IL-10 and its removal enhances the chlamydial M1 pro-inflammatory phenotype (Figures 6(u) and 6(w)). Contrastingly, chlamydial stimulants and ISO induced low mrcl expression and removal of the endogenous IL-10 failed to upregulate mrcl and skew chlamydial-stimulated macrophages to an M2 phenotype (Figures 6(v) and 6(x)). Addition of neutralizing Ab to stimulants with added IL-10 validates the significance of IL-10 in skewing the M1 phenotype (Figures 6(u)-6(x)). Our results provide a compelling role for IL-10 (endogenous and exogenous) in regulating chlamydial macrophage functions and exerting its anti-inflammatory effects. 
IL-6

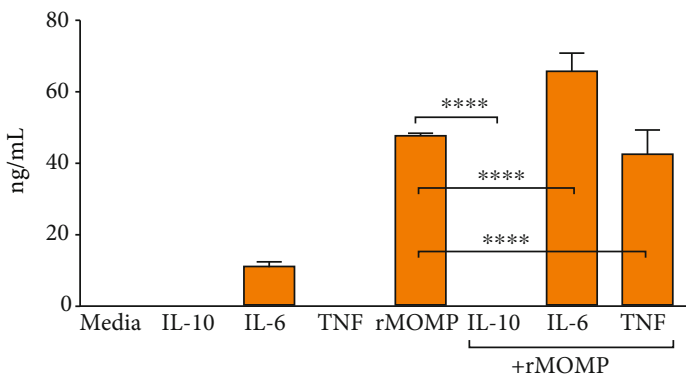

(a)

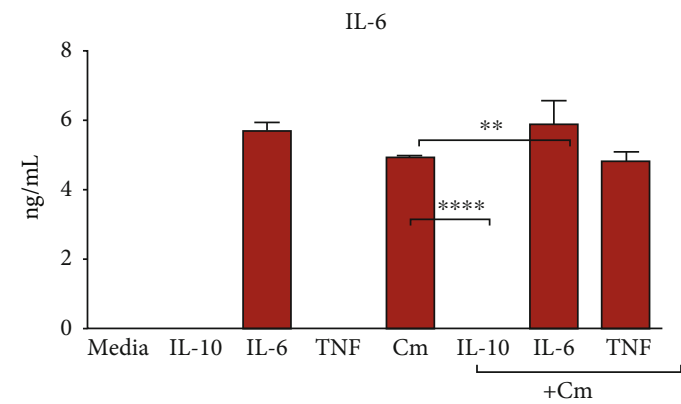

(c)

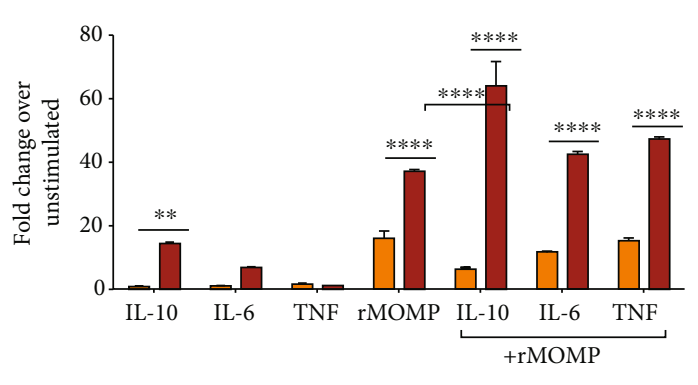

口 SOCS1

- SOCS3

(e)

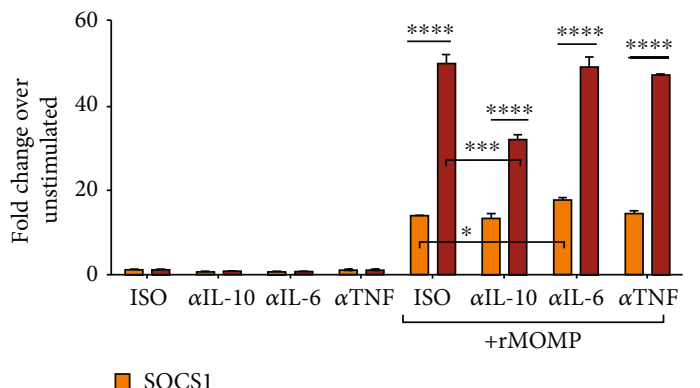

$\square$ SOCS1

- SOCS3

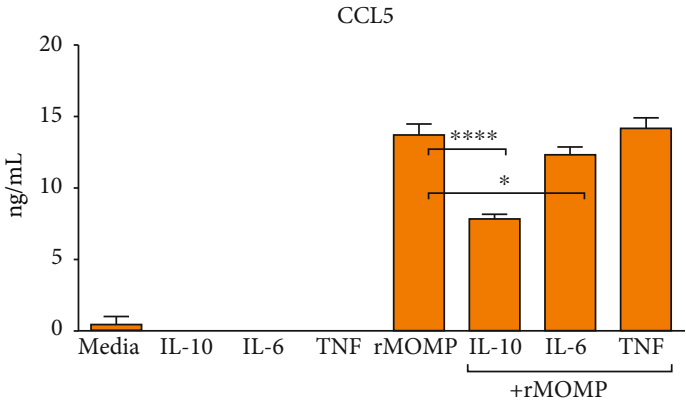

(b)

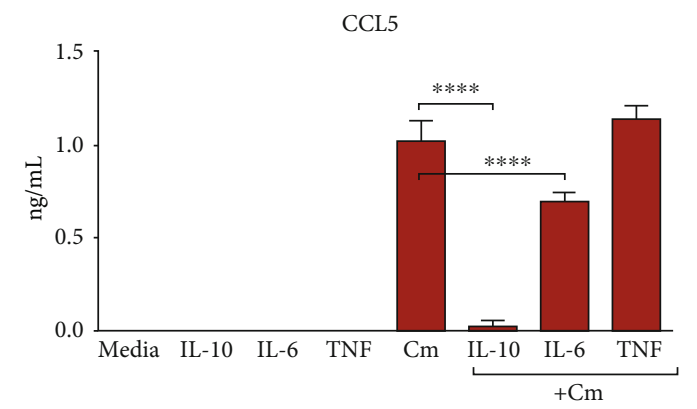

(d)

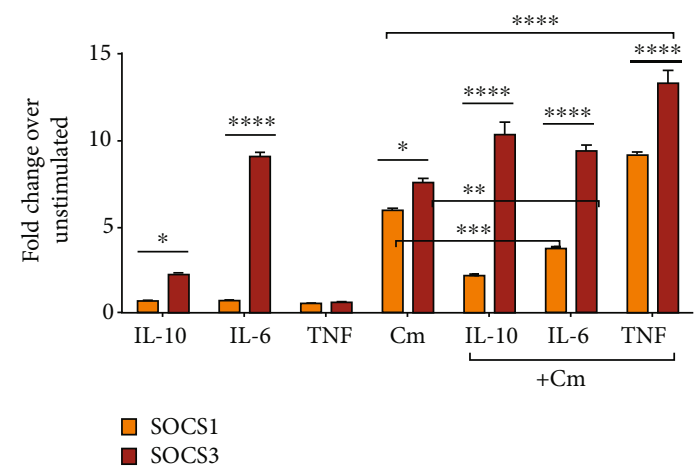

(f)

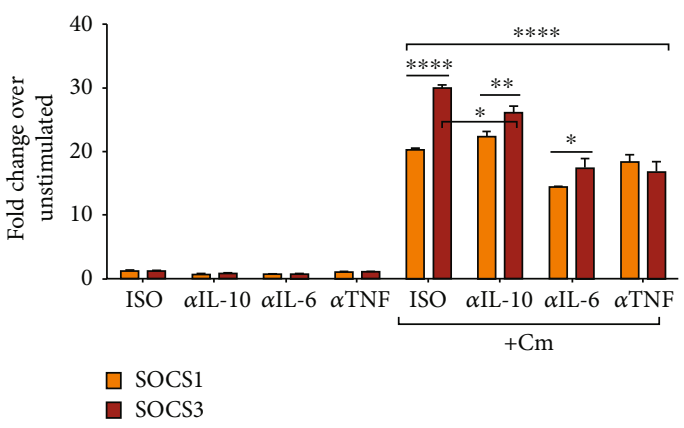

(h)

FIGURE 5: The effect of exogenous and endogenously produced IL-6 and TNF on the expression of inflammatory mediators and SOCS in chlamydial-stimulated macrophages. Macrophages $\left(10^{6} / \mathrm{mL}\right)$ were stimulated with rMOMP $(10 \mu \mathrm{g} / \mathrm{mL})$ or Cm $(\mathrm{MOI}$ of 2$)$ in the presence and absence of IL-10, IL-6, and TNF (each at $10 \mathrm{ng} / \mathrm{mL}$ ) for $24 \mathrm{~h}$. RNA and cell-free supernatants were collected to quantify IL-6 and CCL5 (A-D) along with SOCS1 and SOCS3 mRNA gene transcripts (E-F), respectively, by TaqMan qRT-PCR and specific ELISAs. Macrophages were pre-incubated with neutralizing Abs to IL-10, IL-6, and TNF (each at $25 \mu \mathrm{g} / \mathrm{mL}$ ) for 30 min before adding rMOMP or $\mathrm{Cm}$ for an additional $24 \mathrm{~h}$. Normal rat IgG1 Ab served as the isotype control (ISO). RNA was collected to quantify SOCS1 and SOCS3 mRNA gene transcripts $(\mathrm{G}-\mathrm{H})$. An asterisk indicates a significant difference $(P<0.05)$, and $P$ values were calculated as described in Figure 1. Each bar represents the mean \pm SD of samples run in triplicates. Each experiment was repeated at least 3 times. 


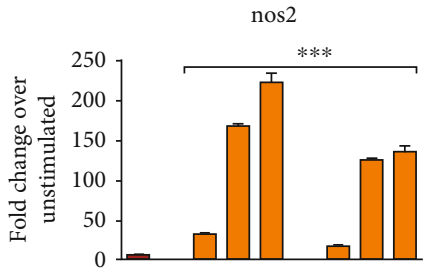

(a)

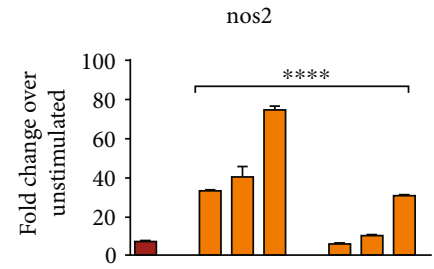

(d)

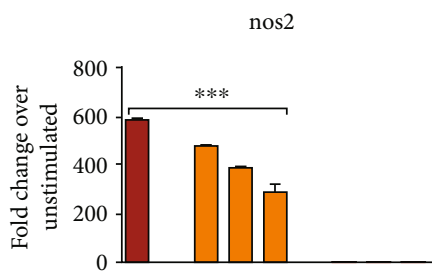

(g)

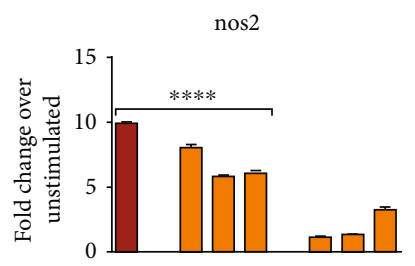

(j)

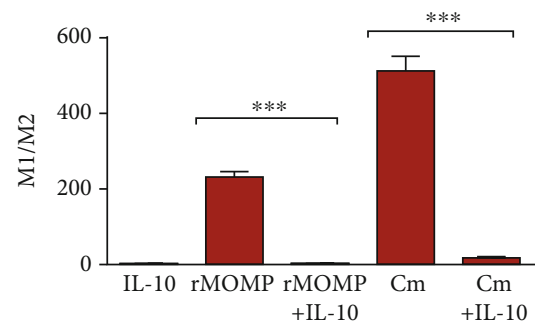

(m)

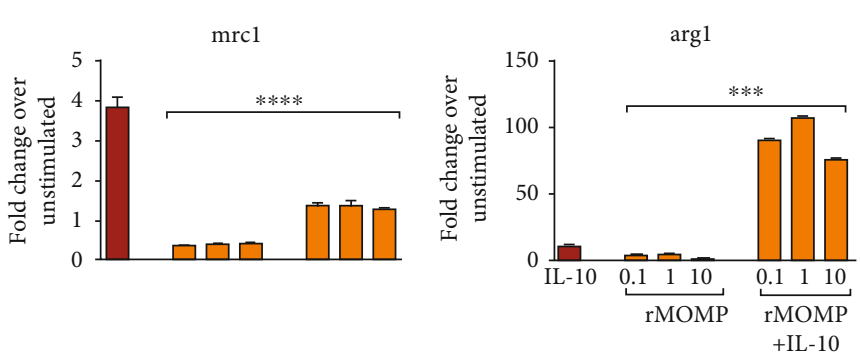

(b)

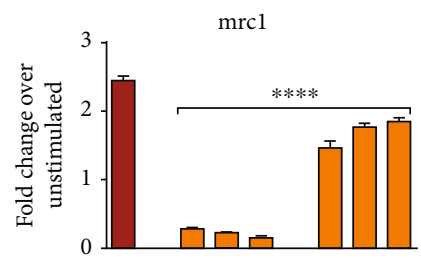

(e)

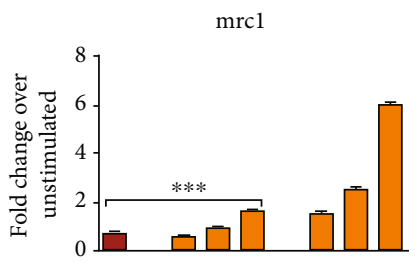

(h)
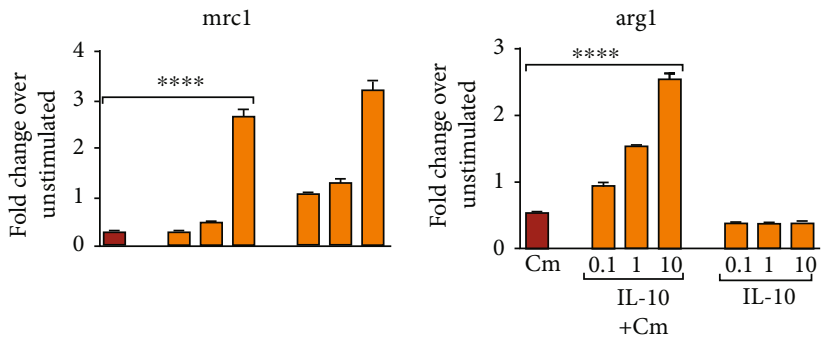

(k)

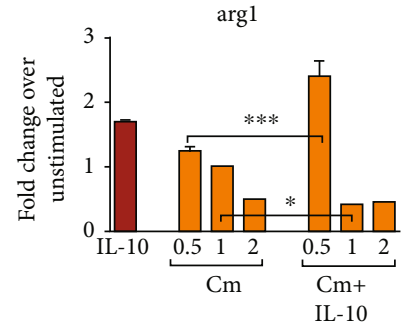

(f)

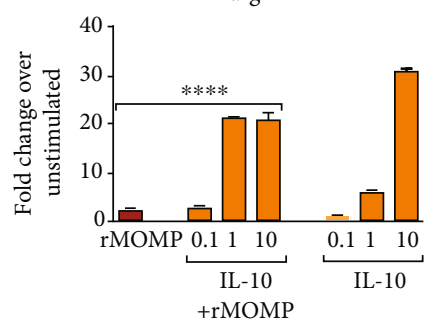

(i)

(l)

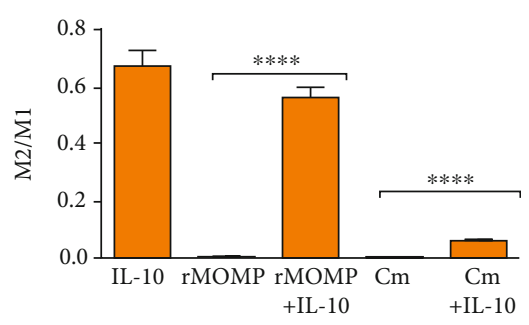

(n)

Figure 6: Continued. 


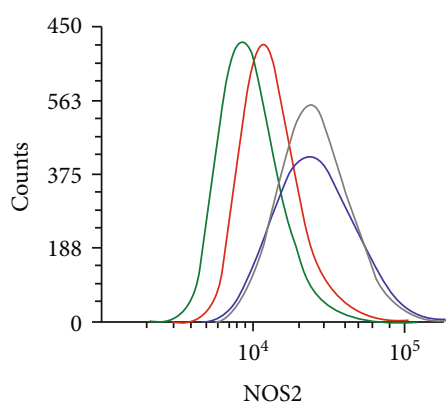

- Cells - IL-10 $_{\text {rMOMP }}$

(o)

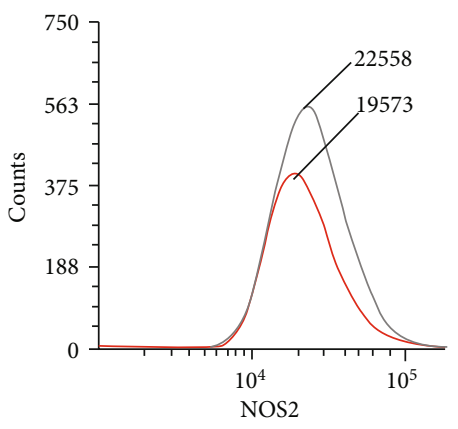

- $\mathrm{Cm}$

(q)

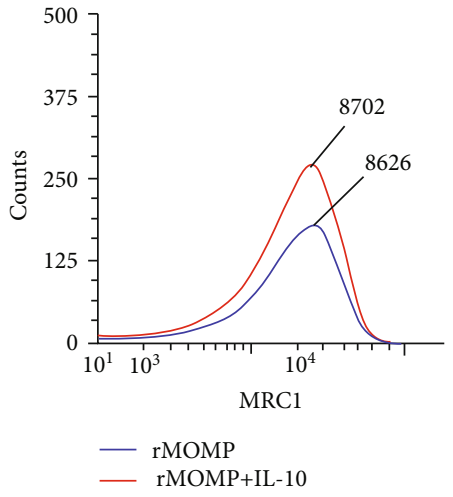

(s)

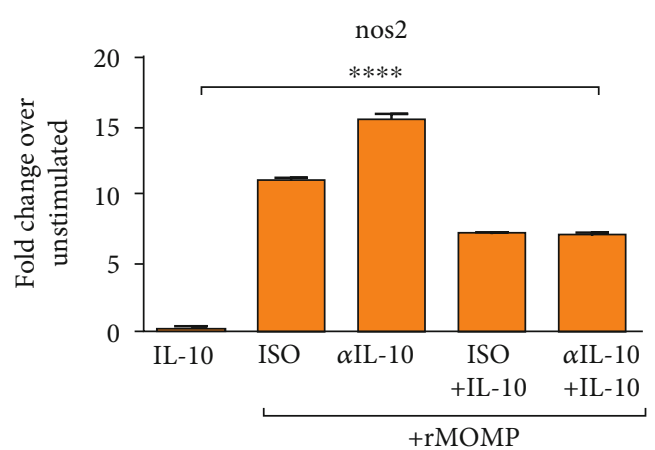

(u)

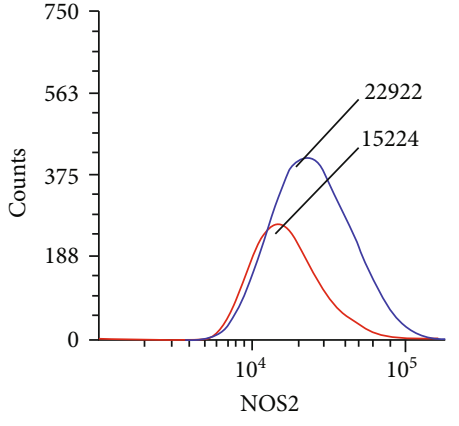

- rMOMP

rMOMP+IL-10

(p)

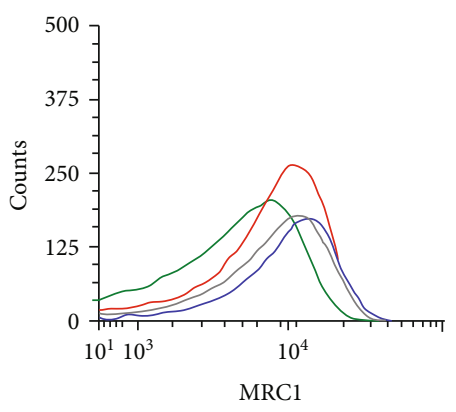

$\begin{array}{ll}\text { - Cells } & \text { IL-10 } \\ \text { rMOMP } & \text { Cm }\end{array}$

(r)

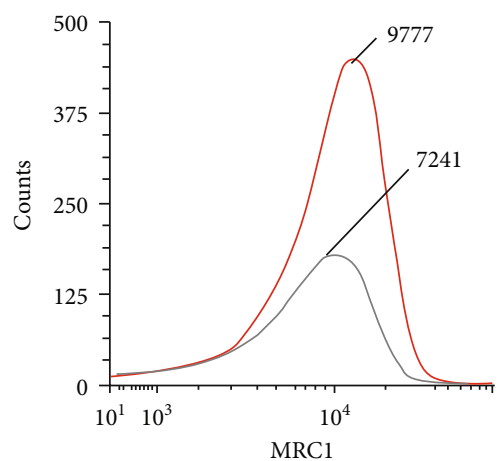

- $\mathrm{Cm}$

_ Cm+IL-10

(t)

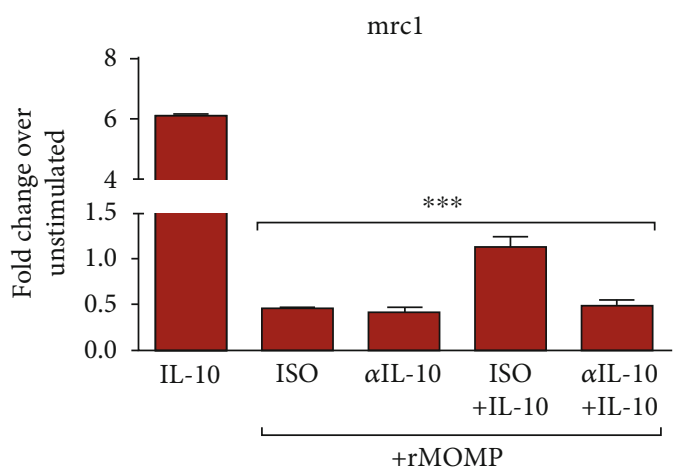

(v)

Figure 6: Continued. 


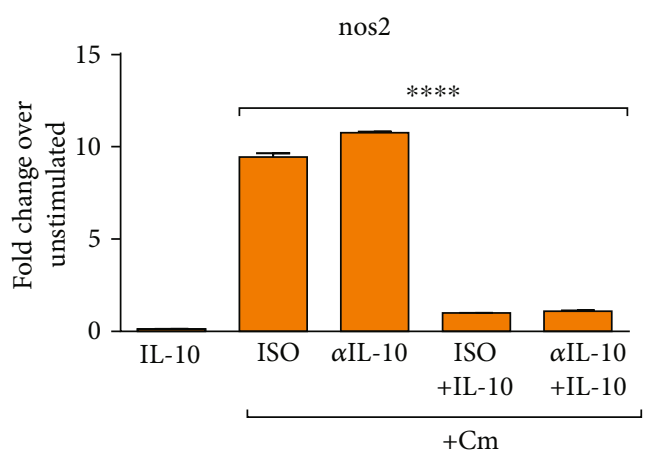

(w)

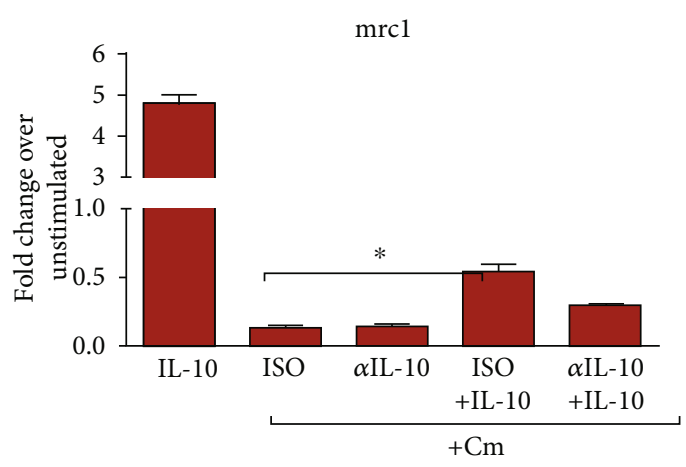

$(\mathrm{x})$

FIGURE 6: Skewing of the chlamydial M1 pro-inflammatory phenotype to an M2 anti-inflammatory phenotype by exogenous and endogenously produced IL-10. Macrophages $\left(10^{6}\right.$ cells $\left./ \mathrm{mL}\right)$ were stimulated with $\mathrm{rMOMP}(0.1,1$ and $10 \mu \mathrm{g} / \mathrm{mL})(\mathrm{A}-\mathrm{C})$ or infected with $\mathrm{Cm}$ (MOI of $0.5,1$ and 2) (D-F) in the presence and absence of IL-10 (10 ng/mL). Macrophages were stimulated with rMOMP (1 $\mu \mathrm{g} / \mathrm{mL}$; G-I) or Cm (MOI of 2; J-L) in the presence and absence of IL-10 $(0.1,1$, and $10 \mathrm{ng} / \mathrm{mL})$. At $24 \mathrm{~h}$ post-stimulation, the mRNA transcripts of the $\mathrm{m} 1$ marker; nos 2 and $\mathrm{m} 2$ markers; $\arg 1$ and $\mathrm{mrc} 1$ were quantified using TaqMan qRT-PCR. The $\mathrm{m} 1 / \mathrm{m} 2(\mathrm{M})$ and $\mathrm{m} 2 / \mathrm{m} 1(\mathrm{~N}) \mathrm{ratios}$ were calculated from macrophages exposed to rMOMP or $\mathrm{Cm}$ in the presence and absence of IL-10. Protein expressions of nos 2 and mrc1 were evaluated from stimulated cells employing flow cytometry (O-T). Macrophages pre-incubated with neutralizing Abs to IL-10, IL-6, and TNF were stimulated with rMOMP or Cm for an additional $24 \mathrm{~h}$. Normal rat IgG1 Ab served as the isotype control (ISO). Poststimulation, RNA was isolated to quantify the gene transcripts of nos2 and mrc1 (U-X) by TaqMan qRT-PCR. An asterisk indicates significant differences $(P<0.05)$, and $P$ values were calculated as described in Figure 1 . Each bar represents the mean \pm SD of samples run in triplicates, and each experiment was repeated at least 3 to 4 times with a representative experiment shown.

3.7. Polarization of M1 and M2 Phenotypes at the Translational Level by Exogenous IL-10 in ChlamydiaStimulated Macrophages. Because IL-10 stimulated an M2 phenotype, we conducted more studies to ensure the fate of chlamydial M1 phenotype in an established M1 and M2 polarizing microenvironment. Reportedly, M1 macrophages are induced by IFN- $\gamma$ or PAMPs, and M2 macrophages by IL-4 and IL-13 [70]. For this purpose, macrophages were pre-incubated for $1 \mathrm{~h}$ with IFN- $\gamma$ to express a proinflammatory M1 phenotype or with IL-4 or IL-13 for a more reparative M2 phenotype, followed by incubation with chlamydial stimulants with and without IL-10. Using TNF as an inflammation marker, we first demonstrated the effect of polarization on TNF induction by chlamydial stimulants. As shown in Figures 7(a)-7(b), polarized macrophages did not secrete TNF. Also, IFN- $\gamma$ polarized macrophages did not alter TNF levels in rMOMP cultures but significantly enhanced $(P<0.0001)$ that of $\mathrm{Cm}$, suggesting stimulation by other chlamydial antigens. A reduction in TNF was seen when chlamydial stimulants and IL-10 were added to IFN- $\gamma$ polarized macrophages, indicating the IL-10 antiinflammatory actions. Conversely, IL-4 polarized macrophages significantly $(P<0.0001)$ down-regulated TNF in chlamydial cultures and when combined with IL-10 for an enhanced additive anti-inflammatory effect.

Further, we quantified M1 and M2 phenotypes of polarized macrophages, including those polarized by IL-13 and IL-10 and only for rMOMP. As depicted in Figure 7(c), nos2 mRNA gene transcripts were not significantly induced by IL4, IL-10, nor IL-13 polarized macrophages either alone or combined. Both IFN- $\gamma$-polarized macrophages and rMOMP alone stimulated marked expression of nos 2 and when co-cultured, increased nos 2 expressions by 14 to 28 -fold, suggesting a synergistic enhancement of the M1 pro-inflammatory phenotypes. Noted are that polarized IL-10, IL-4 and IL-13 macrophages co-cultured with rMOMP reduced nos2 expression; but only the polarized IL-4 and IL-13 macrophages reduced nos 2 expression as induced by IFN $-\gamma$.

Opposing results were attained for $\mathrm{mrcl}$ where polarization of macrophages with IL-10, IL-4 and IL-13, but not IFN- $\gamma$, significantly increased $(P<0.0001)$ expression of mrcl alone or when IL-10 was delivered with IL-4 or IL-13, suggesting the selective stimulation of the M2 phenotype by these polarizing cytokines (Figure $7(\mathrm{~d})$ ). Only IL-4polarized macrophages significantly skewed $(P<0.001)$ the M1 phenotype of IFN- $\gamma$ (up to 2-fold), suggesting an efficient IL-4 polarization. Contrastingly, polarized IL-4, IL-10, and IL-13 macrophages exposed to rMOMP significantly enhanced $(P<0.0001) \mathrm{mrcl}$ expression (up to 5-fold), which indicates skewing of an M1 phenotype to an M2 phenotype. These results disclose and corroborate the skewing of the chlamydial pro-inflammatory M1 phenotype towards a repressive inflammatory M2 phenotype, not only by IL-10 polarization, but also by IL- 4 and IL-13, especially IL- 4 with its potent anti-inflammatory activity in stimulated macrophages. Moreover, the data highlights that polarization of macrophage functions and phenotypes by IL-10 is multifaceted and dependent on the stimulating microenvironment.

Analysis of the SOCS1/SOCS3 ratios of polarized macrophages revealed that the IL-10 SOCS1/SOCS3 ratio was low as compared to the IL- 4 and IL-13 M2 phenotypes, further indicating differential induction of SOCS by these cytokines (Figure $7(\mathrm{e})$ ). Delivery of IL-10 to polarized IL-4 or IL-13 macrophages decreased their SOCS1/SOCS3 ratios, implying that IL-10 regulates their M2 phenotypes. The rMOMPinduced SOCS1/SOCS3 ratio in contrast to that of FN- $\gamma$ 


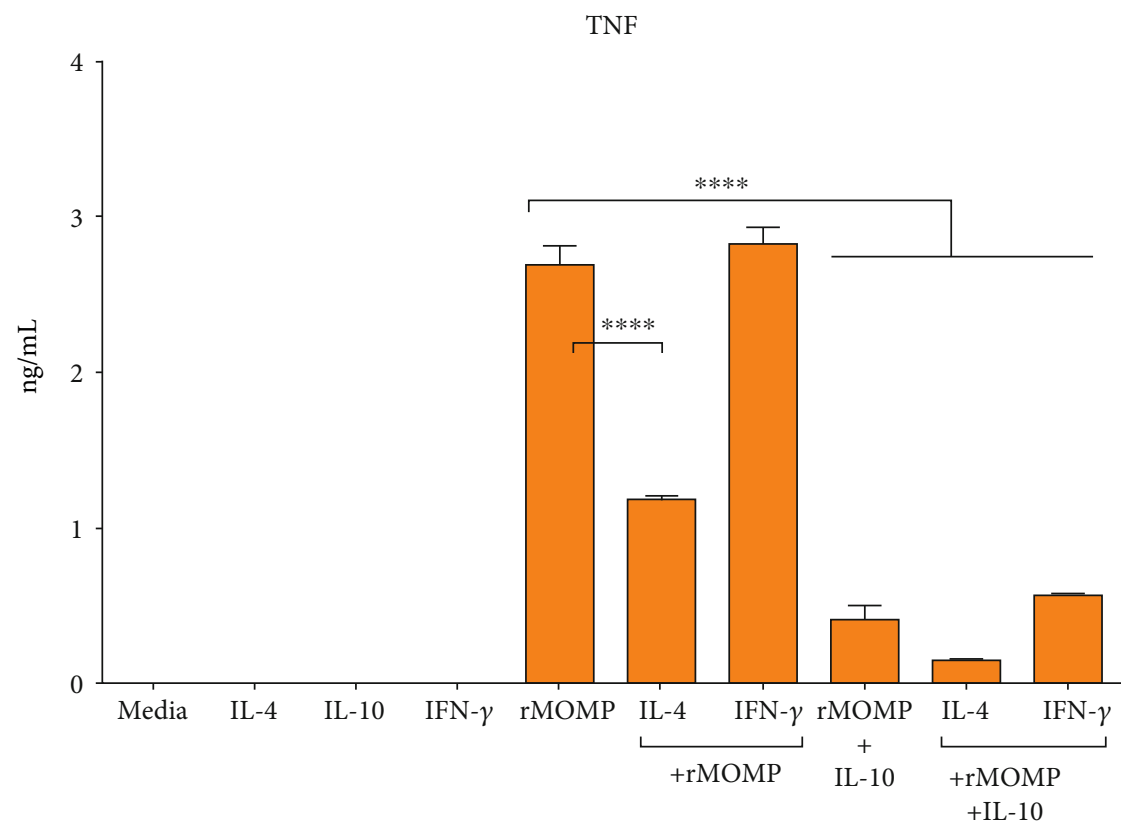

(a)

TNF

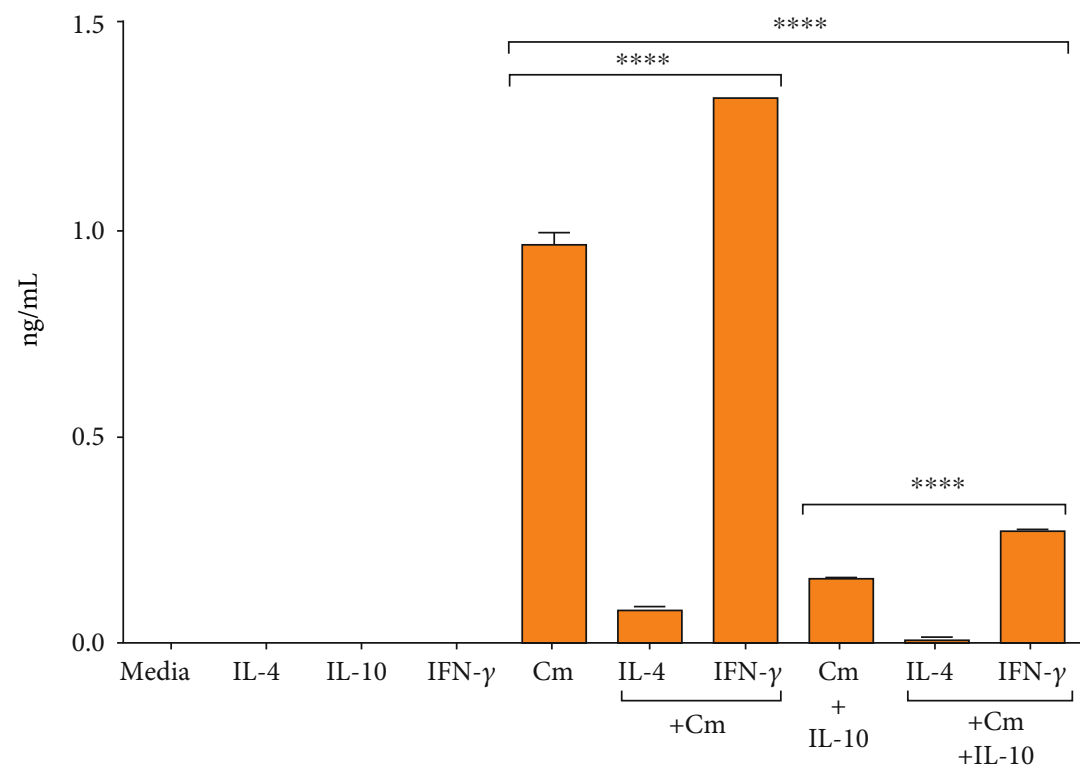

(b)

Figure 7: Continued. 


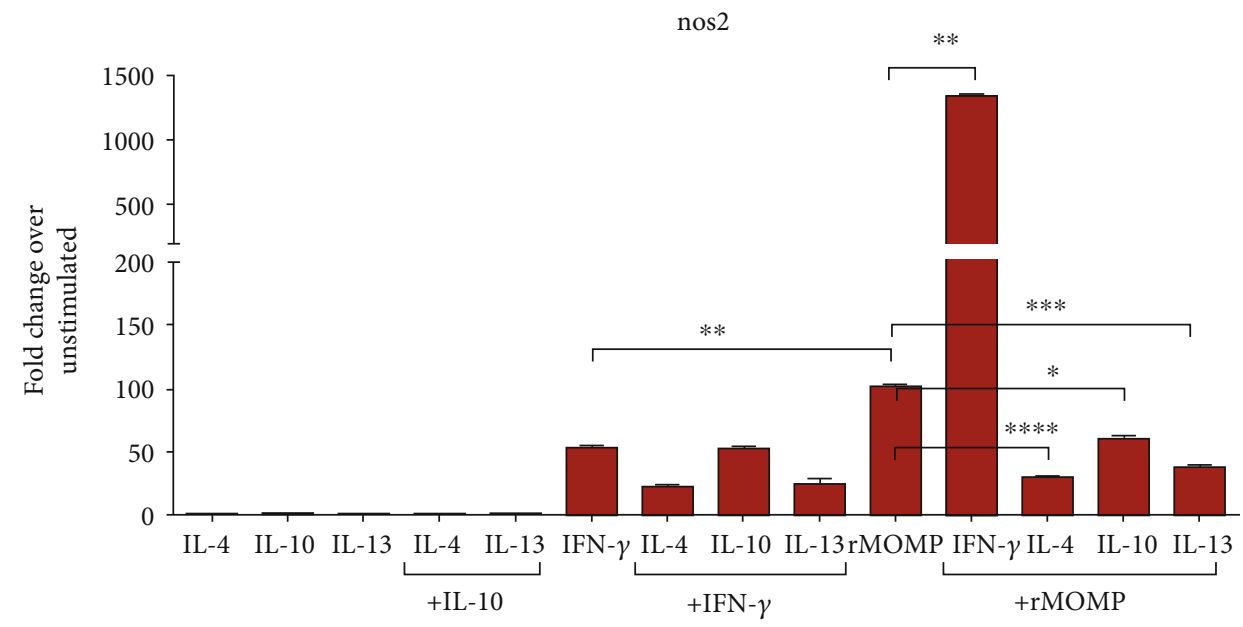

(c)

$\operatorname{mrcl}$

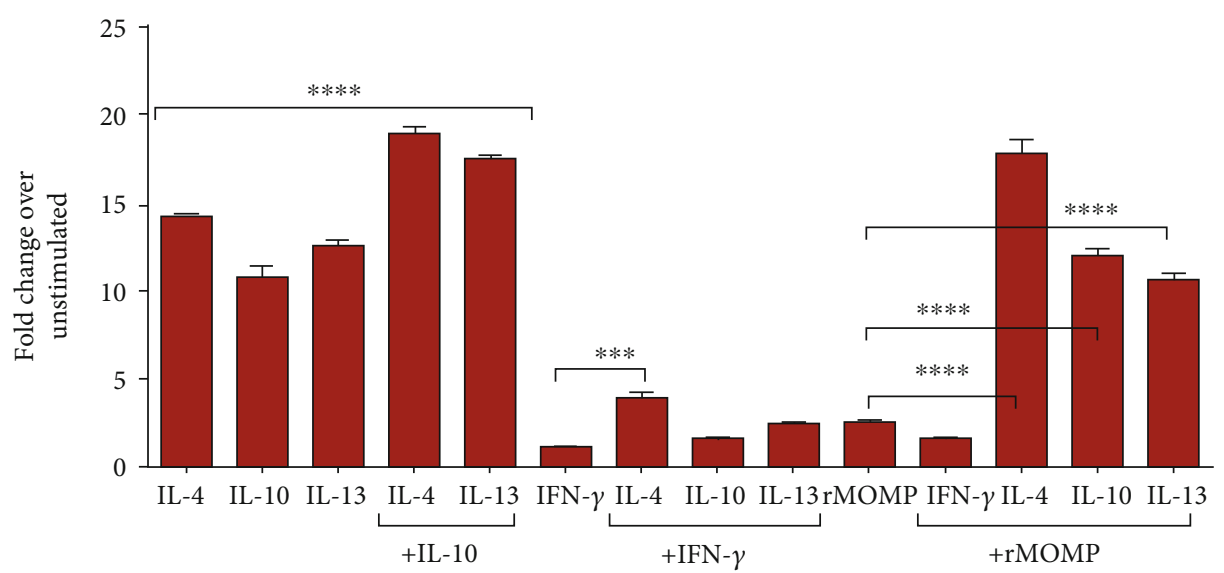

(d)

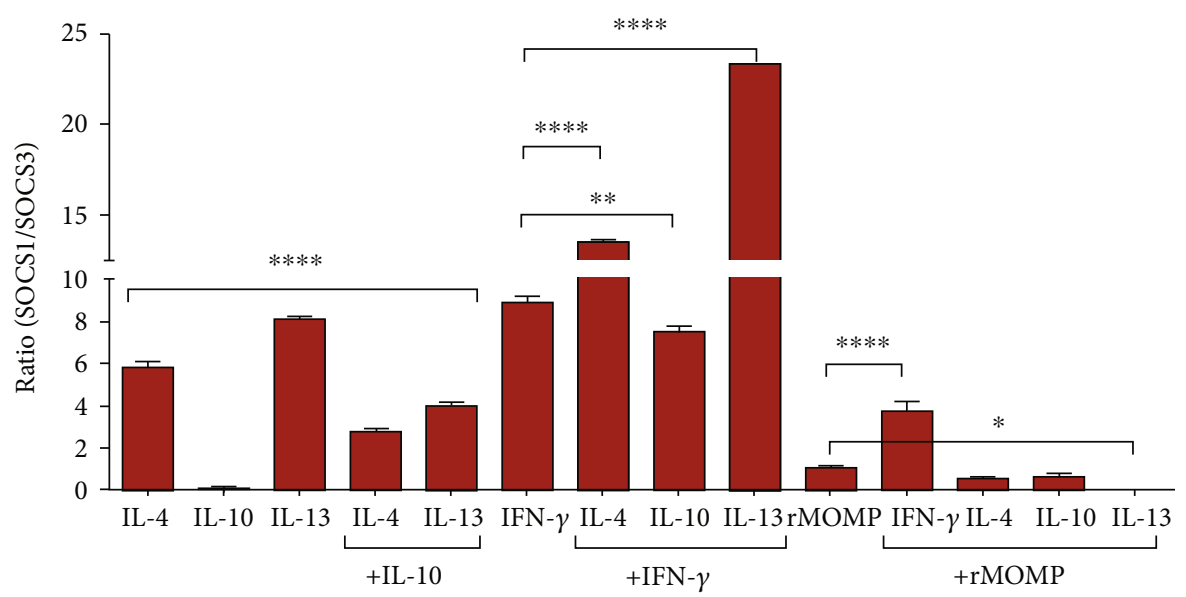

(e)

FIGURE 7: Impact of macrophage polarization on TNF expression, macrophage phenotypes as well as SOCS ratios in rMOMP cultures. Macrophages $\left(10^{6} / \mathrm{mL}\right.$ ) were pre-incubated with IL-4 or IFN- $\gamma$ (each at $10 \mathrm{ng} / \mathrm{mL}$ ) for $1 \mathrm{~h}$ followed by stimulation with either Cm or its rMOMP in the presence and absence of IL-10 for an additional $24 \mathrm{~h}$. Cell-free culture supernatants were collected to quantify TNF by ELISA (A-B). Macrophages were pre-incubated with IL-4, IL-10, IL-13, or IFN- $\gamma$ for $1 \mathrm{~h}$ followed by stimulation with rMOMP for an additional $24 \mathrm{~h}$. The mRNA gene transcripts for nos2, mrc1, socs1 and socs3 (C-E) were quantified via TaqMan qRT-PCR. An asterisk indicates significant differences $(P<0.05)$, and $P$ values were calculated as described in Figure 1 legend. Each bar represents the mean \pm SD of samples run in triplicates, and each experiment was repeated at least 3 to 4 times with a representative experiment shown. 
was lower, implying higher SOCS1 induction by IFN- $\gamma$. Notably, IL-4 and IL-13 polarization increased the IFN- $\gamma$ induced SOCS1/SOCS3 ratio, while the ratio was reduced by IL-10, suggesting that IL-10 differentially regulates SOCS expression compared to the other M2 polarizing cytokines. IL-10, IL-4, and IL-13 polarization reduced the SOCS1/SOCS3 ratio stimulated by rMOMP; conversely, IFN- $\gamma$ polarized macrophages increased the ratio, which again is indicative of IFN- $\gamma$ inducing a high SOCS1 expression [71]. Our results confirm the polarizing role of IL-10 similarly to IL-4 and IL-13 but with differential regulation of SOCS1 and SOCS3 by their respective M2 polarized phenotypes.

3.8. The Proteasome Inhibitor Bortezomib Alters the AntiInflammatory Effect of Exogenous IL-10 in ChlamydialStimulated Macrophages and Regulates the Expression SOCS and STATs. It is well-established that SOCS1 and SOCS3 proteins mediate proteasomal degradation and ubiquitination through the ubiquitin-proteasome system, which is essential to controlling host innate and adaptive immune responses. We tested whether the IL-10-mediated downregulation of inflammatory mediators is dependent on SOCS-mediated proteasome degradation by conducting experiments employing the proteasome inhibitor, Bortezomib (Btzb). Our results indicate that Btzb (at $20 \mathrm{nM}$ ) enhanced TNF levels in unstimulated and IL-10 cultures, suggesting blockade of TNF from proteasomal degradation (Figure 8(a)). Incubation of chlamydial stimulants with Btzb (at 1 and $20 \mathrm{nM}$ ) increased TNF production and reversed the IL-10-inhibition of TNF release in macrophages. In comparison, Btzb (at $20 \mathrm{nM}$ ) inhibited the endogenously produced IL-10 by rMOMP and with a negligible effect on an already low Cm-induced IL-10, a finding corroborating Btzbinhibition of IL-10 production [72] (Figure 8(b)). Also observed is that Btzb suppressed the expression of CCL5 and CXCL10 in chlamydial macrophages, which were further suppressed by IL-10, suggesting that the IL-10-mediated inhibition of chemokines operates independently of proteasome degradation (Figures 8(c) and 8(d)). The results from this study are evidence to suggest that IL-10 inhibition of inflammatory mediators is multifaceted, and inhibition of proteasome degradation may be more efficient for the antiinflammatory actions of IL-10 against cytokines than chemokines in chlamydial-stimulating macrophages.

To better understand the Btzb-mediated IL-10 regulation of inflammation, we elucidated the putative connections with SOCS1 and SOCS3 expression in macrophages. Btzb significantly enhanced $(P<0.01)$ the IL-10-patterned reciprocal upregulation of SOCS1 and SOCS3, which indicates that both are usual targets for proteasome degradation in an environment containing IL-10. Interestingly, in a chlamydial stimulating macrophage environment, Btzb increased SOCS1 (rMOMP only) and SOCS3 expression, correlating with its inhibition of the endogenously produced IL-10, and further illustrating SOCS1 and SOCS3 differential regulation by IL10. Of utmost significance, Btzb exerted a significant inhibitory effect on SOCS1 (rMOMP only) and SOCS3 expression in chlamydial and IL-10 co-cultures, suggesting the Btzb- mediated IL-10 regulation of inflammation is mediated via the expression of SOCS1 and SOCS3 (Figure 8(e)).

Lastly, Figure 8(f) data discloses that IL-10, as expected, induced enhanced STAT3 than STAT1 expression, and both were significantly increased $(P<0.001)$ after Btzb treatment. Not surprising, the chlamydial M1 macrophages exhibited increased STAT1 expression than STAT3, and both were enhanced by Btzb treatment. With added IL-10 to chlamydial cultures, STAT1 decreased with a corresponding increase in STAT3 expression, indicating their regulation by IL-10. However, with Btzb treatment of co-cultures, an upregulation of STAT1 with a concomitant decrease in STAT3 was observed, suggesting a Btzb dysregulation of STAT1 and STAT3. These results provide new revelations that the IL10-mediated inhibition of cytokine and chemokines is mediated by SOCS1 and SOCS3 via proteasome degradationdependent and independent pathways.

\section{Discussion}

Chlamydia immunopathology is responsible for the shortand long-term complications associated with the persistence of the disease due to its inflammatory nature. Many of the severe complications result from excessive immune responses at the site of infection; a tactic used by the bacterium to weaken host immune responses $[6,27,73]$. Effective antibiotics treatment is available, but the eradication of genital chlamydial diseases has been a challenge due to its asymptomatic nature $[2,26,74]$. Moreover, current therapeutic and preventive strategies that promote clearance of bacteria-mediated immune responses may also indirectly intensify pro-inflammatory responses, thereby exacerbating tissue damage $[75,76]$. Su et al. and Batteiger et al. studies revealed that the accelerated eradication of genital Chlamydia by doxycycline also increased the population's susceptibility to reinfection by hindering the development of protective immunity $[77,78]$. The asymptomatic nature of Chlamydia makes an identification, therapy, and prevention of sequelae a challenge [79]. As such, there is an urgency for an approved vaccine or alternative immune intervention strategy that can modulate inflammation while limiting pathology during the early infection. Previously, we reported that IL-10 is an efficacious regulator of Chlamydia-induced inflammatory responses in macrophages [27]. Here, several observations were made in deciphering the mechanisms of IL-10 inhibition of these inflammatory responses. These include 1) MOMP as a mediator of Chlamydia disease pathogenesis; 2) chlamydial stimulants triggered SOCS1 and SOCS3, but with more SOCS3 expression. IL-10 reciprocally regulated their expression by reducing SOCS1 and increasing SOCS3; 3) IL-10 (exogenous and endogenously produced) inhibited chlamydial inflammatory responses through the differential induction and regulation of SOCS1 and SOCS3 proteins; 4) the p38, JNK and MEK1/2 MAPK pathways are regulators of chlamydial inflammatory responses and SOCS1 and SOCS3; 5) chlamydial stimulants triggered an M1 proinflammatory phenotype that was skewed by IL-10 to an M2 anti-inflammatory phenotype to mediate its inhibitory actions, and 6) inhibition of proteasome degradation altered 


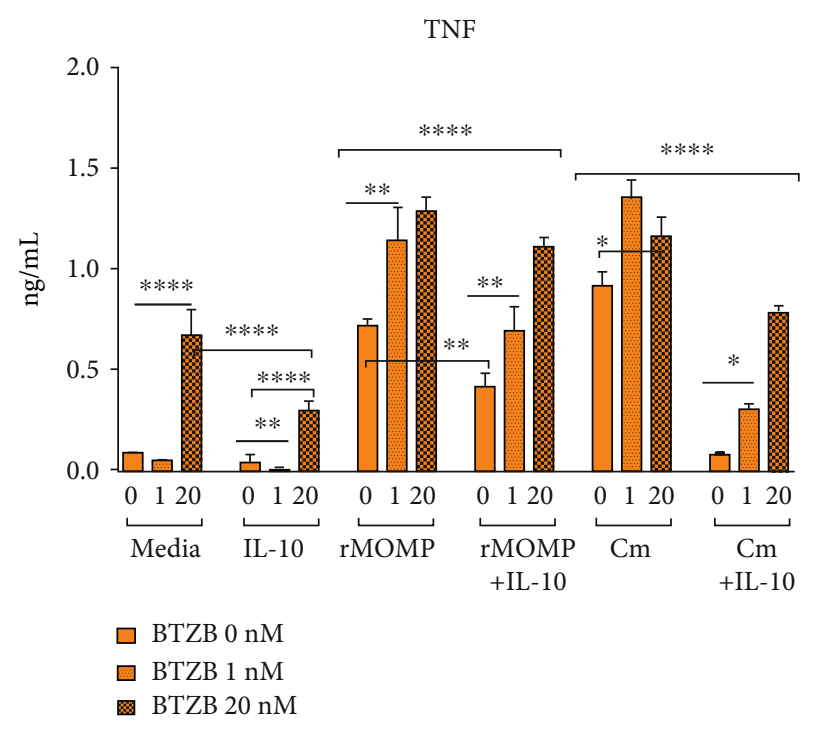

(a)

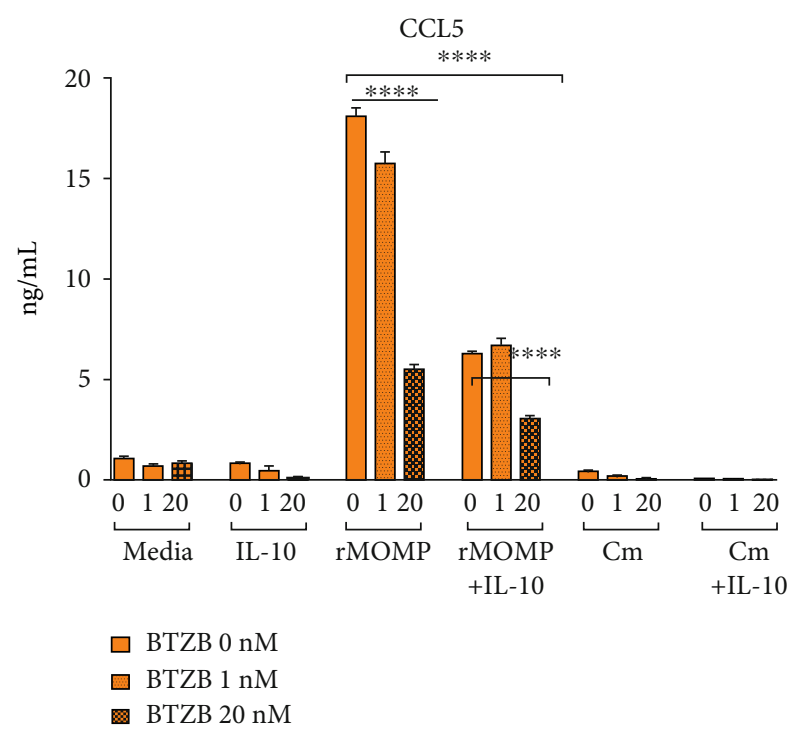

IL - 10

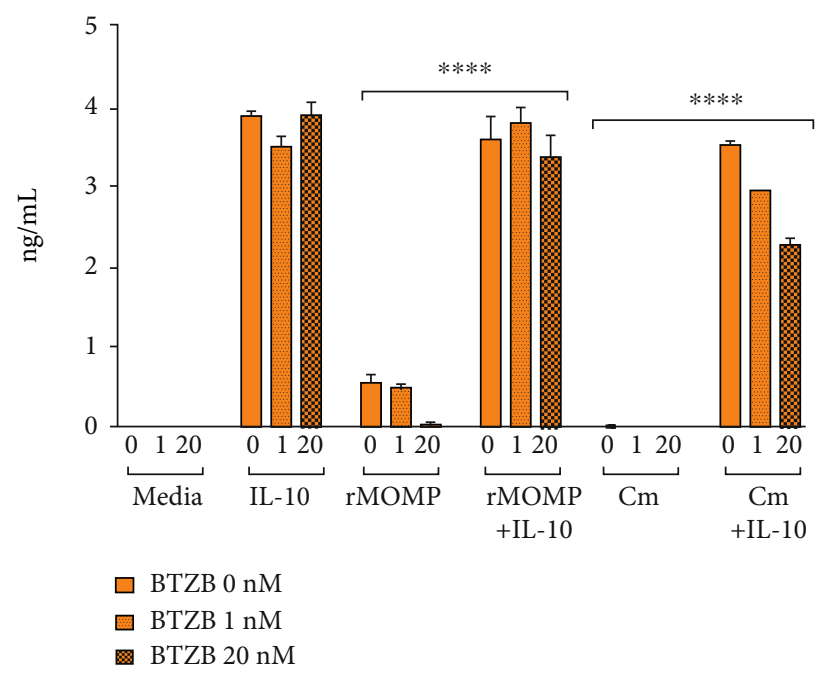

(b)

CXCL10

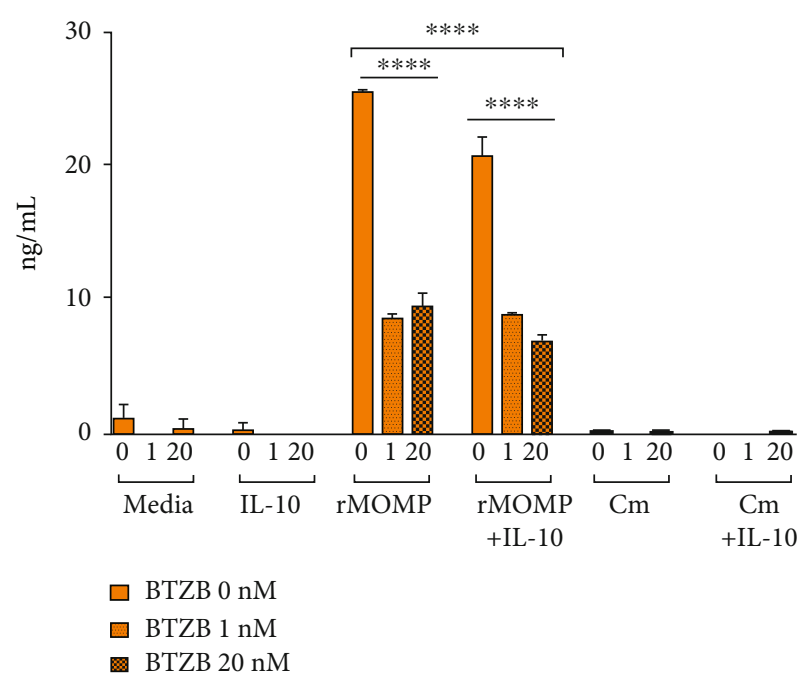

(d)

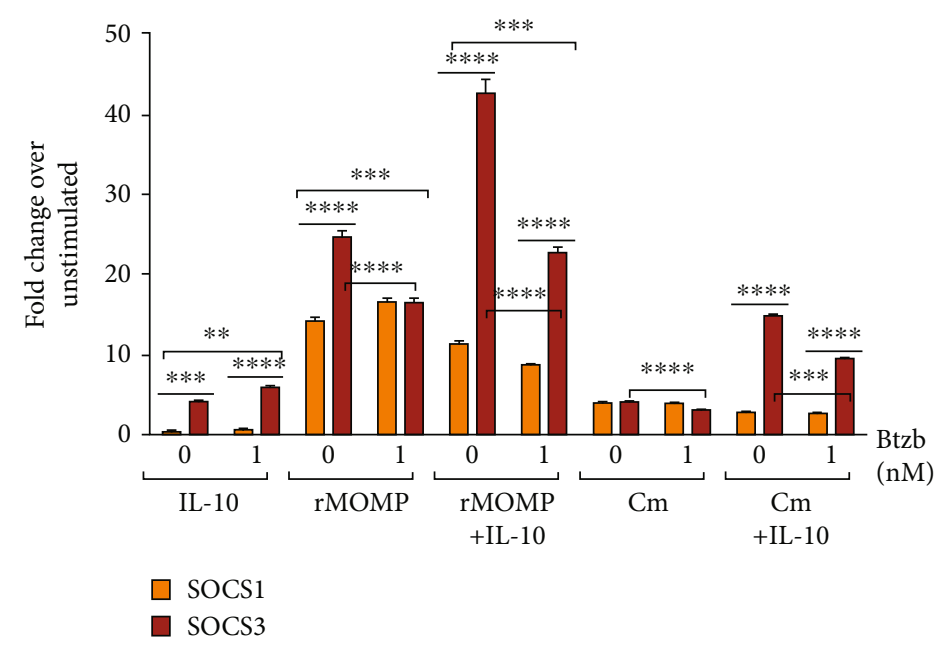

(e)

FIgURE 8: Continued. 


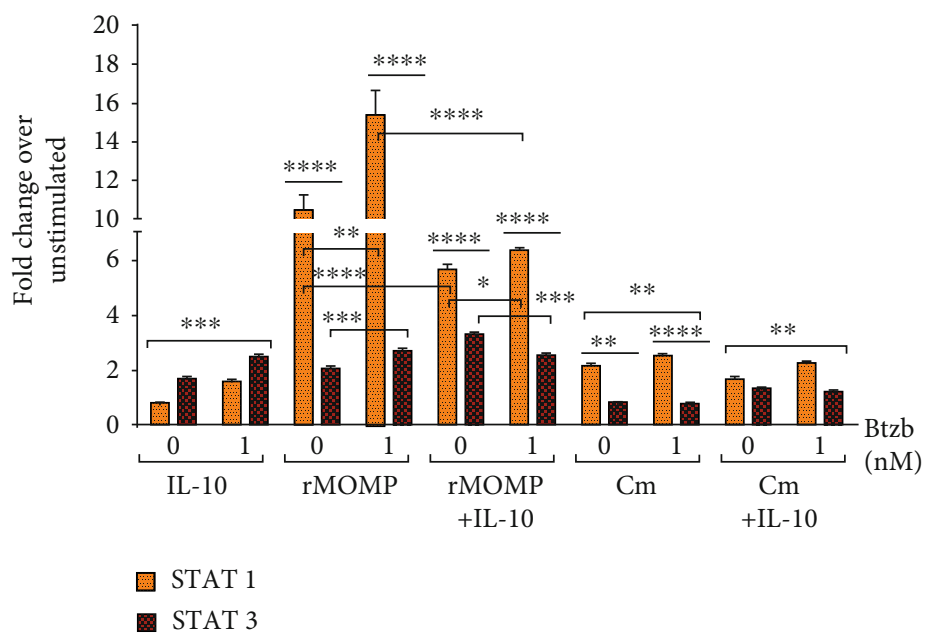

(f)

FIGURE 8: Inhibition of proteasomal degradation alters the IL-10 inhibition of inflammatory mediators and the transcriptional activation of SOCS and STATs in chlamydial macrophage cultures. Macrophages $\left(10^{6} / \mathrm{mL}\right)$ were treated with 0,1 , and $20 \mathrm{nM}$ of Bortezomib (Btzb) for $1 \mathrm{~h}$, followed by stimulation with rMOMP $(1 \mu \mathrm{g} / \mathrm{mL})$ or Cm (MOI of 2$)$ in the presence and absence of IL-10 for $24 \mathrm{~h}$. Post stimulation, cell-free culture supernatants were collected to quantify TNF (A), IL-10 (B), CCL5 (C) and CXCL10 (D) by specific ELISAs. The mRNA gene transcripts for socs1 and socs3 (E) and stat1 and stat3 (F) were quantified via TaqMan qRT-PCR. An asterisk indicates a significant difference $(P<0.05)$, and $P$ values were calculated as described in Figure 1 legend. Each bar represents the mean \pm SD of samples run in triplicates. Each experiment was repeated at least 3 times.

the expression of inflammatory mediators by suppressing SOCS1 and SOCS3, and dysregulating their STAT1 and STAT3 transcription factors.

Macrophages responsiveness to Pathogen-associated Molecular Patterns (PAMPs) can perpetuate inflammation, and Chlamydia proficiently infects and persists within macrophages, resulting in the recruitment of multiple innate immune effectors to the infection site [80-82]. Rajaram et al. demonstrated that macrophages play critical roles in innate and adaptive immunity against chlamydial infections [82]. It is well-established that macrophages serve as vehicles for the propagation and persistence of chlamydial elementary and reticulate bodies that are known to enhance its disease pathogenesis [83-87]. IL-10 anti-inflammatory effects on innate immune responses can further be attributed to macrophages and dendritic cells $[88,89]$. Our previous study showed that live Chlamydia induced the secretion of proinflammatory mediators [26, 27], which corroborate reports of Chlamydia and its recombinant macrophage infectivity potentiator (rMip) stimulating the secretion of Interleukin$1 \beta$ (IL- $1 \beta$ ), TNF, IL-6, and IL-8 in infected human macrophages [90]. In the present study, rMOMP induced copious levels of inflammatory mediators, hence identifying MOMP as a mediator of chlamydial pathogenesis and its role in the establishment of early chlamydial infections. A study by Massari and colleagues delineated that MOMP proteosomes stimulated IL-6 and IL-8 secretions in endocervical epithelial cells [91]. Recently, Cheong et al. reported that MOMP triggered the release of pro-inflammatory cytokines in peripheral blood mononuclear cells (PBMCs) from C. trachomatisinfected patients [92]. The complexity of chlamydial diseases demands further investigations into the mechanisms by which chlamydial antigens activate host cell inflammatory responses at the site of colonization and infection for the disease pathogenesis, which are essential for proper insights in developing improved therapeutic strategies.

Herein, the potent IL-10 anti-inflammatory function $[31,93,94]$ is now validated for chlamydial inflammation by impeding the release of not only cytokines but also chemokines in macrophages, which are congruent with our previous findings [27, 41]. Many investigators have reported on IL-10 inhibitory actions in macrophages exposed to protozoans (Leishmania braziliensis and Leishmania chagasi), viruses (Tacaribe Virus), fungal and bacteria (Chlamydia spp., Borrelia burgdorferi, Helicobacter pylori, Staphylococcus aureus, Streptococcus pneumoniae, and Yersinia enterocolitica) [95]. Moreover, both human and murine T cells reactive to Chlamydia heat shock protein (Hsp60) produce IL-10 that can down-regulate Th1 immune responses [96]. Penttilä et al. demonstrated that while accelerated bacterial clearance was observed in IL-10 knock out (KO) mice, the absence of IL-10 inhibitory effect resulted in severe inflammation post C. pneumoniae infection [97], hence highlighting the importance of IL-10 in promoting host clearance of Chlamydia infections while also reducing pro-inflammatory responses. Reportedly, exogenously added IL-10 to infected C57BL/6 IL-10 KO mice down-regulated pro-inflammatory cytokines (IFN- $\gamma$, TNF, and IL-12) during an acute C. pneumoniae infection, which provides supporting evidence of an indispensable role of IL-10 in regulating Chlamydia-induced inflammation [98].

We further disclose that chlamydial simulants secrete IL10 that self-regulates the magnitude of the concomitantly elicited inflammatory mediators. This observation indeed 
signals that Chlamydia might harness the immunosuppressive capacity of IL-10 to limit host initial anti-bacterial immune responses and facilitate its survival, or that reduction of excessive inflammatory mediators by the initial infection may be mediated via IL-10. Interestingly, Du et al. showed that C. trachomatis endogenously produced IL-10 modulated pro-inflammatory cytokines in human PBMCs [99]. This is consistent with a phenomenon where evolutionarily, pathogens can exploit the repressive function of IL-10 for their benefit to establish chronic infections [31, 88]. In this regard, Noto et al. reported that endogenous IL-10 promoted the optimal phagocytic activity of macrophages in vitro, and its administration to Acinetobacter baumanniiinfected mice diminished a fatal outcome [100]. Others have documented that IL-10-deficient mice develop exacerbated fever in response to lipopolysaccharide (LPS) $[101,102]$ that also led to amplified secretion of IL-1 $\beta$, IL-6, and TNF [103]. This highlights the importance of not only the role of IL-10 in immune regulation but also the impact of its dysregulation in many diseases $[88,102]$. In contrast, reports have highlighted IL-10 to have different roles in infection models of the lymphocytic choriomeningitis virus [104], Schistosoma mansoni [105], Mycobacterium tuberculosis [106] and Candida albicans [107] where it impedes control and clearance of these pathogens [87]. While IL-10 may have opposing roles in several diseased situations, its function in chlamydial-induced inflammation holds promise for many therapeutic advantages. Overall, we delineate an encompassing role for IL-10 (endogenous and exogenous) in minimizing the excessive inflammatory responses induced by Chlamydia infections.

Our results reveal that chlamydial stimulants upregulated SOCS1 and SOCS3 in macrophages with higher SOCS3 expression, which underscore our previous findings [41]. Interestingly, endogenously produced IL-6 and TNF triggered by live $\mathrm{Cm}$ contributed to the overall expression of SOCS1 and SOCS3 since their abrogation reduced SOCS1 and SOCS3 expression. This unique observation for live Cm but not rMOMP may imply direct stimulation of SOCS1 and SOCS3 by rMOMP and probably de novo protein synthesis of SOCS by live Cm. The underlying consequence for the induction of SOCS1 and SOCS3 by Chlamydia may be a mechanistic ploy to limit its inflammation during early infection for perpetuation and survival in the host. It is well-known that SOCS1 and SOCS3 are rapidly induced by TLR agonists [108, 109], which in part explain their induction by a variety of bacterial pathogens, including Borrelia burgdorferi [39], Listeria monocytogenes [110], Mycobacterium bovis [111], M. tuberculosis [112], Salmonella enterica [113], and S. aureus [114], or bacterium-derived substances such as LPS $[115,116]$ and CpG-DNA [117]. Our previous [41] and current findings demonstrate that Chlamydia and its MOMP can differentially induce SOCS1 and SOCS3 in macrophages.

The results here elegantly unveiled that IL-10 immunomodulation of chlamydial inflammatory mediators in macrophages is mediated by simultaneously up-regulating SOCS3 and down-regulating SOCS1; thereby promoting SOCS proteins integral role in its anti-inflammatory actions. In this regard, Dennis et al. reported that co-stimulation of J774 macrophages with live spirochetes of B. burgdorferi and IL10 resulted in IL-10 decreasing cytokines expression while enhancing SOCS1 and SOCS3 expression [39], further inferring that SOCS proteins are involved in the IL-10 antiinflammatory effect in macrophages. Of significance, in the current study, neutralization of endogenously produced IL-10 resulted in a significant decrease in SOCS3 expression. The marked increase in inflammatory mediators and the reduced SOCS3 expression due to blockade of endogenous IL-10 supports SOCS3 as a mediator and regulator of IL-10-inhibitory activity in a chlamydial stimulating macrophage environment. This finding suggests that the removal of endogenous IL-10 while it may not impair total SOCS induction, it is essential for reparation of inflammatory responses.

Triggering of MAPK pathways leads to activation of the transcription factor, NFKB, with subsequent downstream induction of a plethora of inflammatory mediators [118], which are the hallmarks of many inflammatory diseases including Chlamydia [19, 26, 27]. Several of these pathways, including p38, MEK1/2, and JNK, regulated IL-10 inhibition of inflammatory mediators as well as the expression of SOCS1 and SOCS3 in our study. Abrogation of these pathways perturbed the immunosuppressive nature of IL-10, as denoted by their regulation of cytokine and chemokine expression. Guimarães et al. demonstrated that the antiinflammatory compound, curcumin inhibited LPS-induced inflammatory cytokines in macrophages via mechanisms that involve modulating the expression and activity of SOCS1, SOCS3, and p38, which underscores our findings [119]. Inhibition of MAPK pathways has been exploited as potential therapeutics for diseases, including Alzheimer's [120] and inflammatory bowel disease [121]. Additionally, preclinical data targeting inhibitors of the p38 and JNK pathways suggest that they exhibit anti-inflammatory effects [122]. Consequently, a better understanding of signaling events will provide insight into the molecular mechanisms of IL-10-mediated reduction of inflammatory responses during an early Chlamydia infection. Given that blocking MAPK signaling pathways offered differential manifestations by both Chlamydia and IL-10, further understanding of tissueand disease-specific regulatory mechanisms for MAPK signaling pathways might provide clues for the development of efficacious anti-chlamydial therapeutics.

Macrophages play an indispensable, anti-pathogenic role in the regulation and resolution of inflammation [123, 124]. An immunological paradigm exists where macrophages can be activated via an M1 and M2 dichotomy to maintain homeostasis within the cell environment [125, 126]. The M1 macrophage phenotype is characterized by the production of high levels of pro-inflammatory responses, whereas M2 macrophages are characterized by their anti-inflammatory activities [127]. Here we provide some evidence for the IL-10-dependent regulation of Chlamydia-induced inflammatory responses in both M1- and M2-activated and M1- and M2-polarized macrophages. Chlamydia stimulants upregulated the nos2, M1 marker, while barely inducing the arg1 and mrc1, M2 markers, coinciding with their pro-inflammatory potent M1 phenotypic 
activity. Our results are congruent with those of Tang et al., who showed that $M$. tuberculosis triggered macrophage polarization towards an M1 phenotype by producing high concentrations of inflammatory mediators, and also increasing M1 cells and decreasing M2 cells in infected patients [128]. Here, IL-10 downregulated nos2 genes and upregulated both $\arg 1 \mathrm{mrcl}$ as induced by Chlamydia stimulants, suggesting its ability to skew macrophage phenotypes from an M1-like environment towards a more healing M2-like environment for its inhibitory actions.

Recent studies have established the potential for SOCS proteins to regulate M1 and M2 macrophage polarization [109]. Supposedly, a high SOCS1 to SOCS3 ratio could be a potential marker for M2 macrophages, while high SOCS3 to SOCS1 ratio is associated with M1 cells in in vivo experiments [109]. Contrastingly, we show that SOCS3, but not SOCS1, is efficiently up-regulated in M2 macrophages, and this rapid increase in SOCS3 may have a significant role in sustaining some features of the anti-inflammatory phenotype. For chlamydial M1 macrophages, SOCS1 is an essential regulator not only of pro-inflammatory mediators but also of IL-10 anti-inflammatory effects and, consequently, may act to prevent overshooting of the inflammatory response. Therefore, we speculate that modulation of SOCS1 expression represents a potential strategy to control imbalanced macrophage activation in inflammatory diseases. Reportedly, both M1-like and M2-like macrophages can be reprogrammed depending on the appropriate stimuli $[125,126]$. In this regard, we found that the M2 activators, IL-4, and IL-10 synergistically decreased nos 2 in chlamydial cultures while enhancing $\mathrm{mrcl}$, highlighting the potential of these potent therapeutic cytokines in inflammatory response resolution. Our findings suggest that individual or combinations of M2 activators can efficiently reverse M1-like phenotypes towards a more M2-like environment to suppress chlamydial acute inflammatory responses. Moreover, because of the impact of M1/M2 activation in moderating host immune responses, identification, and modulation of macrophage phenotypes in inflammatory diseases seem therapeutically useful $[129,130]$.

The threshold, magnitude, and specific responses elicited by cytokine stimulation are regulated by numerous mechanisms, including SOCS proteins-mediated proteasomal degradation [131]. In the present study, Btzb increased TNF but suppressed CCL5 and CXCL10 secretions in chlamydial macrophages, suggesting an inverse relationship between blocking proteasomal degradation of cytokines versus chemokines, and its immunostimulatory and immunosuppressive effects. Our results are congruent with those of Cleophas et al., who demonstrated that Btzb reverses cytokine suppression by the drug romidepsin by increasing IL$1 \beta$ in a patient gout model [132]. Likewise, reports by Sanacora et al. disclose that Btzb treatment increased IL-8 secretion in macrophages and monocytes [133], which supports the notion of additional cytokines as potential targets for proteasome degradation. Other investigators have shown that Btzb treatment down-regulated CXCL9 released by activated $\mathrm{T}$ cells [134], further underscoring our CCL5 and CXCL10 data.
Herein, Btzb treatment of chlamydial macrophages resulted in lower IL-10 secretions and altered IL-10 regulation of SOCS1 and SOCS3, which may account for IL-10 differential anti-inflammatory effects in regulating inflammatory mediators. Btzb suppressed SOCS1 and SOCS3 expression, especially SOCS3 in chlamydial macrophages both alone and co-incubated with IL-10, indicating that perhaps SOCS3 is more susceptible to proteasomal degradation than SOCS1. Noteworthy, our results disclosed that the decreased SOCS expression may be linked to the repression of IL-10 anti-inflammatory activity evidently by the restored TNF release. Interestingly, being that SOCS1 and SOCS3 are negative feedback regulators of STAT1 and STAT3, respectively, we further illustrate that Btzb treatment caused dysregulation of their expression in chlamydial cultures. These observations suggest that blocking of SOCS1 and SOCS3 actions by Btzb impacts STAT activity, which inadvertently influences the immunomodulatory functions of IL-10 in chlamydial inflammation. While there is limited information on the effects of Btzb on SOCS and STAT induction in the progression of chlamydial diseases, our result holds promise for a better understanding of the complexities of SOCS and other inflammatory pathways as utilized by Chlamydia and IL-10 for their opposing effects during an early Chlamydia infection and necessitate further investigations.

In conclusion, our study provides novel insights into the complex role of IL-10 in suppressing chlamydial inflammatory responses and identifies MOMP as a mediator of its disease pathogenesis. Our findings confirm that IL-10 inhibits chlamydial inflammatory responses in macrophages by activating p38-, JNK- and MEK1/2-MAPK dependent pathways coupled with simultaneously skewing chlamydial M1 proinflammatory towards a more reparative M2 phenotype and underscore SOCS1 and SOCS3 as mediators for its inhibitory actions. This data has important therapeutic implications in targeting IL-10 and SOCS in macrophages and, therefore, could be beneficial for controlling Chlamydia and other bacterial inflammatory diseases. Notably, the reciprocal regulation of SOCS1 and SOCS3 in macrophage by PLA-PEG-encapsulated IL-10 to prolong its biological half-life warrants more studies [41]. Our future research encompasses studying the in vivo role of SOCS1 and SOCS3 in the IL-10-mediated inhibition of chlamydial-induced genital inflammation to expound on the roles of these regulators in the remediation of Chlamydia inflammatory etiologies.

\section{Data Availability}

The data used to support the findings of this study are available from the corresponding author upon request.

\section{Conflicts of Interest}

The authors declare that the research was conducted in the absence of any commercial or financial relationships that could be construed as a potential conflict of interest. 


\section{Authors' Contributions}

SAD contributed to the design of the experiments, performed the experiments, analyzed the data, and wrote the manuscript. RS performed and analyzed the flow cytometry data and helped with the immunofluorescence images; SD helped with the SOCS gene transcription studies, SRS read and critically edited the manuscripts; VAD designed the experiments, critically edited the manuscript, and coordinated the project. All authors read and approved the final manuscript.

\section{Acknowledgments}

The authors would like to thank Yvonne Williams, LaShaundria Lucas, and Juwana Smith-Henderson of CNBR for their excellent administrative assistance. Special thanks also go to Ms. McIver and the Ph. D. program in Microbiology at Alabama State University. The research reported in this publication was supported by the National Institute of Allergy and Infectious Diseases of the National Institutes of Health under Award Number R21AI111159, NIH-NIGMS-RISE (2R25GM106995-06A1) and the National Science Foundation (NSF)-CREST (HRD-1241701) and NSF-HBCU-RISE (HRD-1646729) grants. The content of this study is solely the responsibility of the authors and does not necessarily represent the official views of the National Institutes of Health.

\section{References}

[1] K. Owusu-Edusei Jr., H. W. Chesson, T. L. Gift et al., “The estimated direct medical cost of selected sexually transmitted infections in the United States, 2008," Sexually Transmitted Diseases, vol. 40, no. 3, pp. 197-201, 2013.

[2] M. Malhotra, S. Sood, A. Mukherjee, S. Muralidhar, and M. Bala, "Genital Chlamydia trachomatis: an update," The Indian Journal of Medical Research, vol. 138, no. 3, pp. 303316, 2013.

[3] C. M. O'Connell and M. E. Ferone, "Chlamydia trachomatis genital infections,” Microbial Cell, vol. 3, no. 9, pp. 390-403, 2016.

[4] D. G. Tsevat, H. C. Wiesenfeld, C. Parks, and J. F. Peipert, "Sexually transmitted diseases and infertility," American Journal of Obstetrics and Gynecology, vol. 216, no. 1, pp. 19, 2017.

[5] C. Mitchell and M. Prabhu, "Pelvic inflammatory disease: current concepts in pathogenesis, diagnosis and treatment," Infectious Disease Clinics of North America, vol. 27, no. 4, pp. 793-809, 2013.

[6] K. A. Redgrove and E. A. McLaughlin, "The role of the immune response in Chlamydia trachomatis infection of the male genital tract: a double-edged sword," Frontiers in Immunology, vol. 5, p. 534, 2014.

[7] G. R. Burstein, C. A. Gaydos, M. Diener-West, M. R. Howell, J. M. Zenilman, and T. C. Quinn, "Incident Chlamydia trachomatis infections among inner-city adolescent females," JAMA, vol. 280, no. 6, pp. 521-526, 1998.

[8] A. A. Azenabor, J. Cintrón-Cuevas, H. Schmitt, and V. Bumah, "Chlamydia trachomatis induces antiinflammatory effect in human macrophages by attenuation of immune mediators in Jurkat T-cells," Immunobiology, vol. 216, no. 12, pp. 1248-1255, 2011.

[9] J. U. Igietseme and A. Murdin, "Induction of protective immunity against Chlamydia trachomatis genital infection by a vaccine based on major outer membrane proteinlipophilic immune response-stimulating complexes," Infection and Immunity, vol. 68, no. 12, pp. 6798-6806, 2000.

[10] C. D. Cambridge, S. R. Singh, A. B. Waffo, S. J. Fairley, and V. A. Dennis, "Formulation, characterization, and expression of a recombinant MOMP Chlamydia trachomatis DNA vaccine encapsulated in chitosan nanoparticles," International Journal of Nanomedicine, vol. 8, pp. 1759-1771, 2013.

[11] S. J. Fairley, S. R. Singh, A. N. Yilma et al., "Chlamydia trachomatis recombinant MOMP encapsulated in PLGA nanoparticles triggers primarily $\mathrm{T}$ helper 1 cellular and antibody immune responses in mice: a desirable candidate nanovaccine," International Journal of Nanomedicine, vol. 8, pp. 2085-2099, 2013.

[12] R. Verma, R. Sahu, S. Dixit et al., "The Chlamydia M278 major outer membrane peptide encapsulated in the poly(lactic acid)-poly(ethylene glycol) Nanoparticulate selfAdjuvanting delivery system protects mice against a Chlamydia muridarum genital tract challenge by stimulating robust systemic and local mucosal immune responses," Frontiers in Immunology, vol. 9, article 2369, 2018.

[13] M. J. Burton, R. L. Bailey, D. Jeffries, D. C. W. Mabey, and M. J. Holland, "Cytokine and fibrogenic gene expression in the conjunctivas of subjects from a Gambian community where trachoma is endemic," Infection and Immunity, vol. 72, no. 12, pp. 7352-7356, 2004

[14] N. Faal, R. L. Bailey, I. Sarr, H. Joof, D. C. W. Mabey, and M. J. Holland, "Temporal cytokine gene expression patterns in subjects with trachoma identify distinct conjunctival responses associated with infection," Clinical and Experimental Immunology, vol. 142, no. 2, pp. 347-353, 2005.

[15] H. Sellami, N. Said-Sadier, A. Znazen, R. Gdoura, D. M. Ojcius, and A. Hammami, "Chlamydia trachomatis infection increases the expression of inflammatory tumorigenic cytokines and chemokines as well as components of the Tolllike receptor and NF- $\kappa \mathrm{B}$ pathways in human prostate epithelial cells," Molecular and Cellular Probes, vol. 28, no. 4, pp. 147-154, 2014.

[16] H. Zhou, Q. L. Huang, Z. Y. Li et al., "PORF5 plasmid protein of Chlamydia trachomatis induces MAPK-mediated proinflammatory cytokines via TLR2 activation in THP-1 cells," Science China. Life Sciences, vol. 56, no. 5, pp. 460-466, 2013.

[17] T. Belay, F. O. Eko, G. A. Ananaba et al., "Chemokine and chemokine receptor dynamics during genital chlamydial infection," Infection and Immunity, vol. 70, no. 2, pp. 844850, 2002.

[18] S. Menon, K. Alexander, P. Timms, J. A. Allan, and W. M. Huston, "CXCL10, CXCL11, HLA-A and IL-1 $\beta$ are induced in peripheral blood mononuclear cells from women with Chlamydia trachomatis related infertility," Pathogens and Disease, vol. 74, no. 1, article ftv099, 2015.

[19] A. N. Yilma, S. R. Singh, L. Morici, and V. A. Dennis, "Flavonoid naringenin: a potential immunomodulator for Chlamydia trachomatis inflammation," Mediators of Inflammation, vol. 2013, Article ID 102457, 13 pages, 2013.

[20] R. S. Stephens, "The cellular paradigm of chlamydial pathogenesis," Trends in Microbiology, vol. 11, no. 1, pp. 44-51, 2003. 
[21] T. Darville and T. J. Hiltke, "Pathogenesis of genital tract disease due to Chlamydia trachomatis," The Journal of Infectious Diseases, vol. 201, Suppl 2, pp. 114-125, 2010.

[22] N. B. Kiviat, P. Wølner-Hanssen, D. A. Eschenbach et al., "Endometrial histopathology in patients with cultureproved upper genital tract infection and laparoscopically diagnosed acute salpingitis," The American Journal of Surgical Pathology, vol. 14, no. 2, pp. 167-175, 1990.

[23] T. A. Skwor, B. Atik, R. P. Kandel, H. K. Adhikari, B. Sharma, and D. Dean, "Role of secreted conjunctival mucosal cytokine and chemokine proteins in different stages of trachomatous disease," PLoS Neglected Tropical Diseases, vol. 2, no. 7, article e264, 2008.

[24] S. Dessus-Babus, T. L. Darville, F. P. Cuozzo, K. Ferguson, and P. B. Wyrick, "Differences in innate immune responses (in vitro) to HeLa cells infected with nondisseminating serovar E and disseminating serovar L2 of Chlamydia trachomatis," Infection and Immunity, vol. 70, no. 6, pp. 3234-3248, 2002.

[25] J. Marrazzo and R. Suchland, "Recent advances in understanding and managing Chlamydia trachomatis infections," F1000Prime Reports, vol. 6, p. 120, 2014.

[26] A. N. Yilma, S. R. Singh, S. Dixit, and V. A. Dennis, "Antiinflammatory effects of silver-polyvinyl pyrrolidone (AgPVP) nanoparticles in mouse macrophages infected with live Chlamydia trachomatis," International Journal of Nanomedicine, vol. 8, pp. 2421-2432, 2013.

[27] A. N. Yilma, S. R. Singh, S. J. Fairley, M. A. Taha, and V. A. Dennis, "The anti-inflammatory cytokine, interleukin-10, inhibits inflammatory mediators in human epithelial cells and mouse macrophages exposed to live and UVinactivated Chlamydia trachomatis," Mediators of Inflammation, vol. 2012, Article ID 520174, 10 pages, 2012.

[28] A. J. Kwilasz, P. M. Grace, P. Serbedzija, S. F. Maier, and L. R. Watkins, "The therapeutic potential of interleukin-10 in neuroimmune diseases," Neuropharmacology, vol. 96, Part A, pp. 55-69, 2015.

[29] H. Mollazadeh, A. F. G. Cicero, C. N. Blesso, M. Pirro, M. Majeed, and A. Sahebkar, "Immune modulation by curcumin: the role of interleukin-10," Critical Reviews in Food Science and Nutrition, vol. 59, no. 1, pp. 89-101, 2017.

[30] A. Saxena, S. Khosraviani, S. Noel, D. Mohan, T. Donner, and A. R. A. Hamad, "Interleukin-10 paradox: a potent immunoregulatory cytokine that has been difficult to harness for immunotherapy," Cytokine, vol. 74, no. 1, pp. 27-34, 2015.

[31] J. M. Rojas, M. Avia, V. Martín, and N. Sevilla, "IL-10: a multifunctional cytokine in viral infections," Journal of Immunology Research, vol. 2017, Article ID 6104054, 14 pages, 2017.

[32] W. Ouyang, S. Rutz, N. K. Crellin, P. A. Valdez, and S. G. Hymowitz, "Regulation and functions of the IL-10 family of cytokines in inflammation and disease," Annual Review of Immunology, vol. 29, no. 1, pp. 71-109, 2011.

[33] D. G. Brooks, M. J. Trifilo, K. H. Edelmann, L. Teyton, D. B. McGavern, and M. B. A. Oldstone, "Interleukin-10 determines viral clearance or persistence in vivo," Nature Medicine, vol. 12, no. 11, pp. 1301-1309, 2006.

[34] A. J. Carey, C. K. Tan, and G. C. Ulett, "Infection-induced IL10 and JAK-STAT: a review of the molecular circuitry controlling immune hyperactivity in response to pathogenic microbes," JAK-STAT, vol. 1, no. 3, pp. 159-167, 2014.
[35] D. S. Shouval, J. Ouahed, A. Biswas et al., "Interleukin 10 receptor signaling: master regulator of intestinal mucosal homeostasis in mice and humans," Advances in Immunology, vol. 122, pp. 177-210, 2014.

[36] P. J. Murray, "Understanding and exploiting the endogenous interleukin-10/STAT3-mediated anti-inflammatory response," Current Opinion in Pharmacology, vol. 6, no. 4, pp. 379-386, 2006.

[37] S. A. Duncan et al., "SOCS proteins as regulators of inflammatory responses induced by bacterial infections: a review," Frontiers in Microbiology, vol. 8, article 2431, 2017.

[38] T. Tamiya, I. Kashiwagi, R. Takahashi, H. Yasukawa, and A. Yoshimura, "Suppressors of cytokine signaling (SOCS) proteins and JAK/STAT Pathways," Arteriosclerosis, Thrombosis, and Vascular Biology, vol. 31, no. 5, pp. 980-985, 2011.

[39] V. A. Dennis, A. Jefferson, S. R. Singh, Fø. Ganapamo, and M. T. Philipp, "Interleukin-10 anti-inflammatory response to Borrelia burgdorferi, the agent of Lyme disease: a possible role for suppressors of cytokine signaling 1 and 3," Infection and Immunity, vol. 74, no. 10, pp. 5780-5789, 2006.

[40] M. A. Taha, S. R. Singh, and V. A. Dennis, "Biodegradable PLGA85/15 nanoparticles as a delivery vehicle for Chlamydia trachomatis recombinant MOMP-187 peptide," Nanotechnology, vol. 23, no. 32, p. 325101, 2012.

[41] S. A. Duncan, S. Dixit, R. Sahu et al., "Prolonged release and functionality of Interleukin-10 encapsulated within PLAPEG nanoparticles," Nanomaterials, vol. 9, no. 8, p. 1074, 2019.

[42] A. Gautam, S. Dixit, M. Embers et al., "Different patterns of expression and of IL-10 modulation of inflammatory mediators from macrophages of Lyme disease-resistant and -susceptible mice," PLoS One, vol. 7, no. 9, article e43860, 2012.

[43] K. Wolf, E. Fischer, D. Mead et al., "Chlamydia pneumoniae major outer membrane protein is a surface-exposed antigen that elicits antibodies primarily directed against conformation-dependent determinants," Infection and Immunity, vol. 69, no. 5, pp. 3082-3091, 2001.

[44] Z. Wen, M. A. Boddicker, R. M. Kaufhold et al., "Recombinant expression of Chlamydia trachomatis major outer membrane protein in E. Coli outer membrane as a substrate for vaccine research," BMC Microbiology, vol. 16, no. 1, p. 165, 2016.

[45] S. Zhu, J. Chen, M. Zheng et al., "Identification of immunodominant linear B-cell epitopes within the major outer membrane protein of Chlamydia trachomatis," Acta Biochimica et Biophysica Sinica, vol. 42, no. 11, pp. 771-778, 2010.

[46] Y. Wang, E. A. Berg, X. Feng et al., "Identification of surface-exposed components of MOMP of Chlamydia trachomatis serovar F," Protein Science, vol. 15, no. 1, pp. 122-134, 2006.

[47] M. A. Cassatella, S. Gasperini, C. Bovolenta et al., "Interleukin-10 (IL-10) selectively enhances CIS3/SOCS3 mRNA expression in human neutrophils: evidence for an IL-10induced pathway that is independent of STAT protein activation," Blood, vol. 94, no. 8, pp. 2880-2889, 1999.

[48] L. Williams, L. Bradley, A. Smith, and B. Foxwell, "Signal transducer and activator of transcription 3 is the dominant mediator of the anti-inflammatory effects of IL-10 in human macrophages," Journal of Immunology, vol. 172, no. 1, pp. 567-576, 2003. 
[49] Y. Ding, D. Chen, A. Tarcsafalvi, R. Su, L. Qin, and J. S. Bromberg, "Suppressor of cytokine signaling 1 inhibits IL10-mediated immune responses," Journal of Immunology, vol. 170, no. 3, pp. 1383-1391, 2003.

[50] P. Qasimi, A. Ming-Lum, A. Ghanipour et al., "Divergent mechanisms utilized by SOCS3 to mediate interleukin-10 inhibition of tumor necrosis factor $\alpha$ and nitric oxide production by macrophages," The Journal of Biological Chemistry, vol. 281, no. 10, pp. 6316-6324, 2006.

[51] J. J. Babon and N. A. Nicola, "The biology and mechanism of action of suppressor of cytokine signaling 3," Growth Factors, vol. 30, no. 4, pp. 207-219, 2012.

[52] B. Carow and M. E. Rottenberg, "SOCS3, a major regulator of infection and inflammation," Frontiers in Immunology, vol. 5, p. 58, 2014.

[53] A. Yoshimura, T. Naka, and M. Kubo, "SOCS proteins, cytokine signalling and immune regulation," Nature Reviews Immunology, vol. 7, no. 6, pp. 454-465, 2007.

[54] M. Yamamoto, D. M. Standley, S. Takashima et al., "A single polymorphic amino acid on Toxoplasma gondii kinase ROP16 determines the direct and strain-specific activation of Stat3," The Journal of Experimental Medicine, vol. 206, no. 12, pp. 2747-2760, 2009.

[55] B. Carow, X. qun Ye, D. Gavier-Widén et al., "Silencing suppressor of cytokine signaling-1 (SOCS1) in macrophages improves Mycobacterium tuberculosis control in an interferon-gamma (IFN- $\gamma$ )-dependent manner," The Journal of Biological Chemistry, vol. 286, no. 30, pp. 26873-26887, 2011.

[56] G. E. White, A. Cotterill, M. R. Addley, E. J. Soilleux, and D. R. Greaves, "Suppressor of cytokine signalling protein SOCS3 expression is increased at sites of acute and chronic inflammation," Journal of Molecular Histology, vol. 42, no. 2, pp. 137-151, 2011.

[57] X. Q. Wei, W. Cheng, J. Yan, B. Mei, Z. Huo, and P. Yu, "Expression of SOCS-1, 3 induced by Chlamydia trachomatis-infected Hela229," Zhong Nan Da Xue Xue Bao Yi Xue Ban, vol. 29, no. 6, pp. 639-642, 2004.

[58] T. Yang, P. Stark, K. Janik, H. Wigzell, and M. E. Rottenberg, "SOCS-1 protects against Chlamydia pneumoniae-induced lethal inflammation but hampers effective bacterial clearance," Journal of Immunology, vol. 180, no. 6, pp. 40404049, 2008.

[59] D. W. Hommes, M. P. Peppelenbosch, and S. J. H. van Deventer, "Mitogen activated protein (MAP) kinase signal transduction pathways and novel anti-inflammatory targets," Gut, vol. 52, no. 1, pp. 144-151, 2003.

[60] C. Dong, R. J. Davis, and R. A. Flavell, "MAP kinases in the immune response," Annual Review of Immunology, vol. 20, no. 1, pp. 55-72, 2002.

[61] E. K. Kim and E. J. Choi, "Pathological roles of MAPK signaling pathways in human diseases," Biochimica et Biophysica Acta (BBA) - Molecular Basis of Disease, vol. 1802, no. 4, pp. 396-405, 2010.

[62] B. Sahay, R. L. Patsey, C. H. Eggers, J. C. Salazar, J. D. Radolf, and T. J. Sellati, "CD14 signaling restrains chronic inflammation through induction of p38-MAPK/SOCS-dependent tolerance," PLoS Pathogens, vol. 5, no. 12, article e1000687, 2009.

[63] S. Saccani, S. Pantano, and G. Natoli, "p38-dependent marking of inflammatory genes for increased NF- $\kappa$ B recruitment," Nature Immunology, vol. 3, no. 1, pp. 6975, 2002.

[64] J. J. Baldassare, Y. Bi, and C. J. Bellone, "The role of p38 mitogen-activated protein kinase in IL- $1 \beta$ transcription," Journal of Immunology, vol. 162, no. 9, pp. 5367-5373, 1999.

[65] A. M. Badger, J. N. Bradbeer, B. Votta, J. C. Lee, J. L. Adams, and D. E. Griswold, "Pharmacological profile of SB 203580, a selective inhibitor of cytokine suppressive binding protein/p38 kinase, in animal models of arthritis, bone resorption, endotoxin shock and immune function," The Journal of Pharmacology and Experimental Therapeutics, vol. 279, no. 3, pp. 14531461, 1996.

[66] T. ten Hove, B. van den Blink, I. Pronk, P. Drillenburg, M. P. Peppelenbosch, and S. van Deventer, "Dichotomal role of inhibition of p38 MAPK with SB 203580 in experimental colitis," Gut, vol. 50, no. 4, pp. 507-512, 2002.

[67] C. P. Schneider, M. G. Schwacha, and I. H. Chaudry, "The role of interleukin-10 in the regulation of the systemic inflammatory response following trauma-hemorrhage," Biochimica et Biophysica Acta (BBA) - Molecular Basis of Disease, vol. 1689, no. 1, pp. 22-32, 2004.

[68] P. Stenvinkel, M. Ketteler, R. J. Johnson et al., "IL-10, IL-6, and TNF- $\alpha$ : Central factors in the altered cytokine network of uremia-The good, the bad, and the ugly," Kidney International, vol. 67, no. 4, pp. 1216-1233, 2005.

[69] R. C. Miller, E. Schlaepfer, S. Baenziger et al., "HIV interferes with SOCS-1 and -3 expression levels driving immune activation," European Journal of Immunology, vol. 41, no. 4, pp. 1058-1069, 2011.

[70] S. S. Bohlson, S. D. O'Conner, H. J. Hulsebus, M.-M. Ho, and D. A. Fraser, "Complement, clq, and clq-related molecules regulate macrophage polarization," Frontiers in Immunology, vol. 5, p. 402, 2014.

[71] B. Liu, S. Chen, Y. Guan, and L. Chen, "Type III interferon induces distinct SOCS1 expression pattern that contributes to delayed but prolonged activation of Jak/STAT signaling pathway: implications for treatment nonresponse in HCV patients," PLoS One, vol. 10, no. 7, article e0133800, 2015.

[72] T. P. Chang, V. Poltoratsky, and I. Vancurova, "Bortezomib inhibits expression of TGF- $\beta 1$, IL-10, and CXCR4, resulting in decreased survival and migration of cutaneous T cell lymphoma cells," Journal of Immunology, vol. 194, no. 6, pp. 2942-2953, 2015.

[73] C. M. O'Connell, Y. M. AbdelRahman, E. Green et al., "Tolllike receptor 2 activation by Chlamydia trachomatis is plasmid dependent, and plasmid-responsive chromosomal loci are coordinately regulated in response to glucose limitation by C. trachomatis but not by C. muridarum," Infection and Immunity, vol. 79, no. 3, pp. 1044-1056, 2011.

[74] W. E. Stamm, "Chlamydia trachomatis Infections: progress and problems," The Journal of Infectious Diseases, vol. 179, Supplement 2, pp. S380-S383, 1999.

[75] S. E. Howie, P. J. Horner, A. W. Horne, and G. Entrican, "Immunity and vaccines against sexually transmitted Chlamydia trachomatis infection," Current Opinion in Infectious Diseases, vol. 24, no. 1, pp. 56-61, 2011.

[76] R. C. Brunham and J. Rey-Ladino, "Immunology of Chlamydia infection: implications for a Chlamydia trachomatis vaccine," Nature Reviews Immunology, vol. 5, no. 2, pp. 149-161, 2005. 
[77] H. Su, R. Morrison, R. Messer, W. Whitmire, S. Hughes, and H. D. Caldwell, "The effect of doxycycline treatment on the development of protective immunity in a murine model of chlamydial genital infection," The Journal of Infectious Diseases, vol. 180, no. 4, pp. 1252-1258, 1999.

[78] B. E. Batteiger, F. Xu, R. E. Johnson, and M. L. Rekart, "Protective immunity to Chlamydia trachomatis Genital infection: evidence from human studies," The Journal of Infectious Diseases, vol. 201, Supplement 2, pp. 178-189, 2010.

[79] B. D. Taylor and C. L. Haggerty, "Management of Chlamydia trachomatis genital tract infection: screening and treatment challenges," Infection and Drug Resistance, vol. 4, pp. 19-29, 2011.

[80] E. Gracey, A. Lin, A. Akram, B. Chiu, and R. D. Inman, "Intracellular survival and persistence of Chlamydia muridarum is determined by macrophage polarization," PLoS One, vol. 8, no. 8, article e69421, 2013.

[81] A. Teng, M. I. Cruz-Fisher, C. Cheng et al., "Proteomic identification of immunodominant chlamydial antigens in a mouse model," Journal of Proteomics, vol. 77, pp. 176-186, 2012.

[82] K. Rajaram and D. E. Nelson, "Chlamydia muridarum infection of macrophages elicits bactericidal nitric oxide production via reactive oxygen species and cathepsin B," Infection and Immunity, vol. 83, no. 8, pp. 3164-3175, 2015.

[83] H. Yu, K. P. Karunakaran, X. Jiang, and R. C. Brunham, "Subunit vaccines for the prevention of mucosal infection with Chlamydia trachomatis," Expert Review of Vaccines, vol. 15, no. 8, pp. 977-988, 2016.

[84] J. A. Herweg and T. Rudel, "Interaction of chlamydiae with human macrophages," The FEBS Journal, vol. 283 , no. 4 , pp. 608-618, 2016.

[85] A. T. Y. Yeung, C. Hale, A. H. Lee et al., "Exploiting induced pluripotent stem cell-derived macrophages to unravel host factors influencing Chlamydia trachomatis pathogenesis," Nature Communications, vol. 8, no. 1, article 15013, 2017.

[86] A. K. Murthy, W. Li, and K. H. Ramsey, "Immunopathogenesis of chlamydial infections," Current Topics in Microbiology and Immunology, vol. 412, pp. 183-215, 2018.

[87] L. D. Hazlett, X. Jiang, and S. A. McClellan, "IL-10 function, regulation, and in bacterial keratitis," Journal of Ocular Pharmacology and Therapeutics, vol. 30, no. 5, pp. 373380, 2014.

[88] S. S. Iyer and G. Cheng, "Role of interleukin 10 transcriptional regulation in inflammation and autoimmune disease," Critical Reviews in Immunology, vol. 32, no. 1, pp. 23-63, 2012.

[89] K. N. Couper, D. G. Blount, and E. M. Riley, "IL-10: the master regulator of immunity to infection," Journal of Immunology, vol. 180, no. 9, pp. 5771-5777, 2008.

[90] S. Bas, L. Neff, M. Vuillet et al., "The proinflammatory cytokine response to Chlamydia trachomatis elementary bodies in human macrophages is partly mediated by a lipoprotein, the macrophage infectivity potentiator, through TLR2/TLR1/TLR6 and CD14," Journal of Immunology, vol. 180, no. 2, pp. 1158-1168, 2008.

[91] P. Massari, D. N. Toussi, D. F. Tifrea, and L. M. de la Maza, "Toll-like receptor 2-dependent activity of native major outer membrane protein proteosomes of Chlamydia trachomatis," Infection and Immunity, vol. 81, no. 1, pp. 303-310, 2013.
[92] H. C. Cheong, C. Y. Q. Lee, Y. Y. Cheok et al., "CPAF, HSP60 and MOMP antigens elicit pro-inflammatory cytokines production in the peripheral blood mononuclear cells from genital Chlamydia trachomatis- infected patients," Immunobiology, vol. 224, no. 1, pp. 34-41, 2019.

[93] D. Lobo-Silva, G. M. Carriche, A. G. Castro, S. Roque, and M. Saraiva, "Balancing the immune response in the brain: IL-10 and its regulation," Journal of Neuroinflammation, vol. 13, no. 1, p. 297, 2016.

[94] A. R. Burmeister and I. Marriott, "The Interleukin-10 family of cytokines and their role in the CNS," Frontiers in Cellular Neuroscience, vol. 12, p. 458, 2018.

[95] B. L. Duell, C. K. Tan, A. J. Carey, F. Wu, A. W. Cripps, and G. C. Ulett, "Recent insights into microbial triggers of interleukin-10 production in the host and the impact on infectious disease pathogenesis: Table 1," FEMS Immunology and Medical Microbiology, vol. 64, no. 3, pp. 295-313, 2012.

[96] Y. Yi, X. Yang, and R. C. Brunham, “Autoimmunity to heat shock protein 60 and antigen-specific production of interleukin-10," Infection and Immunity, vol. 65, no. 5, pp. 16691674, 1997.

[97] T. Penttilä, A. Haveri, A. Tammiruusu, J. M. Vuola, R. Lahesmaa, and M. Puolakkainen, "Chlamydia pneumoniae infection in IL-10 knock out mice: accelerated clearance but severe pulmonary inflammatory response," Microbial Pathogenesis, vol. 45, no. 1, pp. 25-29, 2008.

[98] X. Yang, J. Gartner, L. Zhu, S. Wang, and R. C. Brunham, "IL10 gene knockout mice show enhanced Th1-like protective immunity and absent granuloma formation following Chlamydia trachomatis lung infection," Journal of Immunology, vol. 162, no. 2, pp. 1010-1017, 1999.

[99] K. du, M. Zhou, Q. Li, and X. Z. Liu, "Chlamydia trachomatis inhibits the production of pro-inflammatory cytokines in human PBMCs through induction of IL-10," Journal of Medical Microbiology, vol. 67, no. 2, pp. 240-248, 2018.

[100] M. J. Noto, K. W. Becker, K. L. Boyd, A. M. Schmidt, and E. P. Skaar, "RAGE-mediated suppression of Interleukin-10 results in enhanced mortality in a murine model of Acinetobacter baumannii sepsis," Infection and Immunity, vol. 85, no. 3, 2017.

[101] L. R. Leon, W. Kozak, K. Rudolph, and M. J. Kluger, “An antipyretic role for interleukin-10 in LPS fever in mice," The American Journal of Physiology, vol. 276, no. 1, pp. R81R89, 1999.

[102] K. S. Chu, A. N. Schorzman, M. C. Finniss et al., "Nanoparticle drug loading as a design parameter to improve docetaxel pharmacokinetics and efficacy," Biomaterials, vol. 34, no. 33, pp. 8424-8429, 2013.

[103] R. Dantzer, J. C. O'Connor, G. G. Freund, R. W. Johnson, and K. W. Kelley, "From inflammation to sickness and depression: when the immune system subjugates the brain," Nature Reviews Neuroscience, vol. 9, no. 1, pp. 46-56, 2008.

[104] M. Ejrnaes, C. M. Filippi, M. M. Martinic et al., "Resolution of a chronic viral infection after interleukin-10 receptor blockade," The Journal of Experimental Medicine, vol. 203, no. 11, pp. 2461-2472, 2006.

[105] D. van der Kleij, E. Latz, J. F. H. M. Brouwers et al., "A novel host-parasite lipid cross-talk. Schistosomal lysophosphatidylserine activates toll-like receptor 2 and affects immune polarization," The Journal of Biological Chemistry, vol. 277, no. 50, pp. 48122-48129, 2002. 
[106] S. Jang, S. Uematsu, S. Akira, and P. Salgame, "IL-6 and IL-10 induction from dendritic cells in response to Mycobacterium tuberculosis is predominantly dependent on TLR2-mediated recognition," Journal of Immunology, vol. 173, no. 5, pp. 3392-3397, 2004.

[107] M. G. Netea, R. Sutmuller, C. Hermann et al., “Toll-like receptor 2 suppresses immunity against Candida albicans through induction of IL-10 and regulatory T cells," Journal of Immunology, vol. 172, no. 6, pp. 3712-3718, 2004.

[108] H. J. Lee, K. C. Kim, J. A. Han, S. S. Choi, and Y. J. Jung, "The early induction of suppressor of cytokine signaling 1 and the downregulation of toll-like receptors 7 and 9 induce tolerance in costimulated macrophages," Molecules and Cells, vol. 38, no. 1, pp. 26-32, 2015.

[109] H. M. Wilson, "SOCS proteins in macrophage polarization and function," Frontiers in Immunology, vol. 5, p. 357, 2014.

[110] D. Stoiber, S. Stockinger, P. Steinlein, J. Kovarik, and T. Decker, "Listeria monocytogenes modulates macrophage cytokine responses through STAT serine phosphorylation and the induction of suppressor of cytokine signaling 3," Journal of Immunology, vol. 166, no. 1, pp. 466-472, 2001.

[111] K. Imai, T. Kurita-Ochiai, and K. Ochiai, "Mycobacterium bovisbacillus Calmette-Gueérin infection promotes SOCS induction and inhibits IFN- $\boldsymbol{\gamma}$-stimulated JAK/STAT signaling in J774 macrophages," FEMS Immunology and Medical Microbiology, vol. 39, no. 2, pp. 173-180, 2003.

[112] R. K. Pai, M. Convery, T. A. Hamilton, W. H. Boom, and C. V. Harding, "Inhibition of IFN- $\gamma$-Induced class II transactivator expression by a $19-\mathrm{kDa}$ lipoprotein from Mycobacterium tuberculosis: a potential mechanism for immune evasion," Journal of Immunology, vol. 171, no. 1, pp. 175-184, 2003.

[113] K. Uchiya and T. Nikai, "Salmonella pathogenicity island 2dependent expression of suppressor of cytokine signaling 3 in macrophages," Infection and Immunity, vol. 73, no. 9, pp. 5587-5594, 2005.

[114] S. Bertholet, H. L. Dickensheets, F. Sheikh, A. A. Gam, R. P. Donnelly, and R. T. Kenney, "Leishmania donovani-induced expression of suppressor of cytokine signaling 3 in human macrophages: a novel mechanism for intracellular parasite suppression of activation," Infection and Immunity, vol. 71, no. 4, pp. 2095-2101, 2003.

[115] A. Crespo, M. B. Filla, and W. J. Murphy, "Low responsiveness to IFN- $\gamma$, after pretreatment of mouse macrophages with lipopolysaccharides, develops via diverse regulatory pathways," European Journal of Immunology, vol. 32, no. 3, pp. 710-719, 2002.

[116] A. Crespo, M. B. Filla, S. W. Russell, and W. J. Murphy, "Indirect induction of suppressor of cytokine signalling-1 in macrophages stimulated with bacterial lipopolysaccharide: partial role of autocrine/paracrine interferon- $\alpha / \beta$," The Biochemical Journal, vol. 349, no. 1, pp. 99-104, 2000.

[117] A. H. Dalpke, S. Opper, S. Zimmermann, and K. Heeg, "Suppressors of cytokine signaling (SOCS)-1 and SOCS-3 are induced by CpG-DNA and modulate cytokine responses in APCs," Journal of Immunology, vol. 166, no. 12, pp. 70827089, 2001.

[118] M. Cargnello and P. P. Roux, "Activation and function of the MAPKs and their substrates, the MAPK-activated protein kinases," Microbiology and Molecular Biology Reviews, vol. 75, no. 1, pp. 50-83, 2011.

[119] M. R. Guimarães, F. R. M. Leite, L. C. Spolidorio, K. L. Kirkwood, and C. Rossa Jr., "Curcumin abrogates LPS- induced pro-inflammatory cytokines in RAW 264.7 macrophages. Evidence for novel mechanisms involving SOCS-1, -3 and p38 MAPK," Archives of Oral Biology, vol. 58, no. 10, pp. 1309-1317, 2013.

[120] I. Churcher, "Tau therapeutic strategies for the treatment of Alzheimer's disease," Current Topics in Medicinal Chemistry, vol. 6, no. 6, pp. 579-595, 2006.

[121] E. Hollenbach, M. Neumann, M. Vieth, A. Roessner, P. Malfertheiner, and M. Naumann, "Inhibition of p38 MAP kinase- and RICK/NF- $\kappa \mathrm{B}$-signaling suppresses inflammatory bowel disease," The FASEB Journal, vol. 18, no. 13, pp. 1550-1552, 2004.

[122] B. Kaminska, "MAPK signalling pathways as molecular targets for anti-inflammatory therapy—from molecular mechanisms to therapeutic benefits," Biochimica et Biophysica Acta (BBA) - Proteins and Proteomics, vol. 1754, no. 1-2, pp. 253262, 2005.

[123] A. Ariel, I. Maridonneau-Parini, P. Rovere-Querini, J. S. Levine, and H. Mühl, "Macrophages in inflammation and its resolution," Frontiers in Immunology, vol. 3, p. 324, 2012.

[124] T. J. Koh and L. A. DiPietro, "Inflammation and wound healing: the role of the macrophage," Expert Reviews in Molecular Medicine, vol. 13, article e23, 2011.

[125] P. Italiani and D. Boraschi, "From monocytes to M1/M2 macrophages: phenotypical vs. functional differentiation," Frontiers in Immunology, vol. 5, p. 514, 2014.

[126] F. O. Martinez and S. Gordon, "The M1 and M2 paradigm of macrophage activation: time for reassessment," F1000Prime Reports, vol. 6, p. 13, 2014.

[127] Y. Wang, W. Smith, D. Hao, B. He, and L. Kong, "M1 and M2 macrophage polarization and potentially therapeutic naturally occurring compounds," International Immunopharmacology, vol. 70, pp. 459-466, 2019.

[128] Y. Tang, S. C. Hua, G. X. Qin, L. J. Xu, and Y. F. Jiang, "Different subsets of macrophages in patients with new onset tuberculous pleural effusion," PLoS One, vol. 9, no. 2, article e88343, 2014.

[129] T. D. Smith, M. J. Tse, E. L. Read, and W. F. Liu, "Regulation of macrophage polarization and plasticity by complex activation signals," Integrative Biology, vol. 8, no. 9, pp. 946-955, 2016.

[130] A. C. Labonte, A. C. Tosello-Trampont, and Y. S. Hahn, “The role of macrophage polarization in infectious and inflammatory diseases," Molecules and Cells, vol. 37, no. 4, pp. 275-285, 2014.

[131] B. A. Croker, H. Kiu, and S. E. Nicholson, "SOCS regulation of the JAK/STAT signalling pathway," Seminars in Cell \& Developmental Biology, vol. 19, no. 4, pp. 414-422, 2008.

[132] M. C. P. Cleophas, T. O. Crişan, V. Klück et al., "Romidepsin suppresses monosodium urate crystal-induced cytokine production through upregulation of suppressor of cytokine signaling 1 expression," Arthritis Research \& Therapy, vol. 21, no. 1 , p. $50,2019$.

[133] S. Sanacora, J. Urdinez, T. P. Chang, and I. Vancurova, “Anticancer drug bortezomib increases interleukin-8 expression in human monocytes," Biochemical and Biophysical Research Communications, vol. 460, no. 2, pp. 375-379, 2015.

[134] S. T. Pellom Jr., D. F. Dudimah, M. C. Thounaojam, T. J. Sayers, and A. Shanker, "Modulatory effects of bortezomib on host immune cell functions," Immunotherapy, vol. 7, no. 9, pp. 1011-1022, 2015. 